\title{
In situ X-ray Diffraction Investigation of the Crystallisation of Perfluorinated Ce(IV)-based Metal-Organic Frameworks with UiO-66 and MIL-140 architectures
}

\author{
Stephen J. I. Shearan, ${ }^{1}$ Jannick Jacobsen, ${ }^{2}$ Ferdinando Costantino, ${ }^{3}$ Roberto D'Amato, ${ }^{3}$ Dmitri Novikov, ${ }^{4}$ Norbert \\ Stock, ${ }^{2}$ Enrico Andreoli, ${ }^{1}$ Marco Taddei ${ }^{11,5}$ \\ ${ }^{1}$ Energy Safety Research Institute, Swansea University, Fabian Way, Swansea SA1 8EN, UK \\ ${ }^{2}$ Institute of Inorganic Chemistry, Christian-Albrechts-University, Max-Eyth-Str. 2, 24118 Kiel, Germany \\ ${ }^{3}$ Department of Chemistry, Biology and Biotechnologies, University of Perugia, Via Elce di Sotto n. 8, 06123 \\ Perugia, Italy \\ ${ }^{4}$ Deutsches Elektronen-Synchrotron DESY, Notkestraße 85, 22607 Hamburg, Germany \\ ${ }^{5}$ Department of Chemistry and Industrial Chemistry, University of Pisa, Via Giuseppe Moruzzi 13, 56124 Pisa, \\ Italy.Email: marco.taddei@unipi.it
}

\begin{abstract}
We report on the results of an in situ synchrotron powder X-ray diffraction study of the crystallisation in aqueous medium of two recently discovered perfluorinated Ce(IV)-based metal-organic frameworks (MOFs), analogues of the already well investigated Zr(IV)-based UiO-66 and MIL-140A, namely, F4_UiO-66(Ce) and F4_MIL$140 \mathrm{~A}(\mathrm{Ce})$. The two MOFs were originally obtained in pure form in similar conditions, using ammonium cerium nitrate and tetrafluoroterephthalic acid as reagents, and small variations of the reaction parameters were found to yield mixed phases. Here, we investigate the crystallisation of these compounds, varying parameters such as temperature, amount of the protonation modulator nitric acid $\left(\mathrm{HNO}_{3}\right)$ and amount of the coordination modulator acetic acid (AcOH). When only $\mathrm{HNO}_{3}$ is present in the reaction environment, only F4_MIL-140A(Ce) is obtained. Heating preferentially accelerates nucleation, which becomes rate determining below $57^{\circ} \mathrm{C}$. Upon addition of $\mathrm{AcOH}$ to the system, alongside $\mathrm{HNO}_{3}$, mixed-phased products are obtained. $\mathrm{F} 4$ _UiO-66(Ce) is always formed faster and no interconversion between the two phases occurs. In the case of F4_UiO-66(Ce), crystal growth is always the rate determining step. A higher amount of $\mathrm{HNO}_{3}$ favours the formation of F4_MIL-140A(Ce), whereas increasing the amount of $\mathrm{AcOH}$ favours the formation of F4_UiO-66(Ce). Based on the in situ results, a new optimised route to achieving a pure, high quality F4_MIL-140A(Ce) phase in mild conditions $\left(60{ }^{\circ} \mathrm{C}, 1 \mathrm{~h}\right)$ is also identified.
\end{abstract}

\section{Introduction}

Investigating the influence of reaction conditions on the thermodynamics and kinetics of crystallisation of metalorganic frameworks (MOFs) is extremely beneficial to gather information on the relationship between crystal structure and synthetic parameters, to optimise the reaction conditions for up-scaling of synthetic protocols and to aid the design of new materials. ${ }^{[1]}$ Since the majority of MOF syntheses is carried out starting from reagents in solution, the formation of the solid product can be followed in situ employing scattering techniques - such as powder X-ray diffraction (PXRD), ${ }^{[2]}$ small angle X-ray scattering (SAXS), ${ }^{[3]}$ static/dynamic light scattering (SLS/DLS), ${ }^{[4]}-\mathrm{NMR}^{[5]}$ or turbidity measurements. ${ }^{[6]}$ SAXS, SLS/DLS and turbidity are especially suited to study the earliest stages of crystallisation because they can detect particles with subnanometric size, but they provide no information about the crystal structure of the scattering objects. ${ }^{[1 a]}$ NMR has shown to be capable of providing insight into the early stages of crystallisation by looking at time-dependant evolution of the liquid phase, whereby nucleation and crystal growth activation parameters can be obtained by looking at the decrease in signal intensity, i.e. the disappearance of the linker, and treatment of subsequent data with models such as the two-step Gualtieri. ${ }^{[5]}$ On the other hand, PXRD is limited to crystallites having size at least in the nanometre range and is blind to amorphous matter, making it not suitable for studying the earliest stages of crystallisation. ${ }^{[1 \mathrm{a}]}$ However, it provides precious information about the crystalline phases present in the system, allowing to study the evolution of multiphase systems and even to identify possible transient, metastable 
phases. ${ }^{[7]}$ Thanks to its ability to provide insight on both kinetics and thermodynamics, PXRD is the most widespread tool for in situ, time-resolved monitoring of MOF crystallisation. ${ }^{[1]}$

Over the past few years, Ce(IV)-MOFs have frequently appeared in the literature, most often based on the connection of hexanuclear $\left[\mathrm{Ce}_{6} \mathrm{O}_{4}(\mathrm{OH})_{4}\right]$ clusters with polycarboxylate-based linkers of various geometries and symmetries. ${ }^{[8]}$ These MOFs have attracted interest mainly thanks to their redox properties, which distinguish them from their $\mathrm{Zr}$ (IV)-based counterparts and can be exploited for various catalytic processes. ${ }^{[8 d, 9]}$ In addition, they also display interesting photocatalytic properties. ${ }^{[10]} \mathrm{A}$ review on Ce-MOFs has recently been published that summarises the synthetic and structural aspects as well as potential applications. ${ }^{[11]}$ The synthesis of these MOFs is typically carried out in $N, N$-dimethylformamide (DMF)/water mixtures as a reaction medium and in situ XRD studies have been key in revealing how the desired product is completely crystallised within a few minutes. ${ }^{\text {[12] }}$ However, this phase is metastable and, if left in the reaction environment, undergoes dissolution to form Ce(III)formate, having formula $\left[\mathrm{Ce}\left(\mathrm{O}_{2} \mathrm{CH}\right)_{3}\right]$. Formation of $\mathrm{Ce}(\mathrm{III})$-formate was attributed to the reaction between $\mathrm{Ce}(\mathrm{III})$, derived from reduction of $\mathrm{Ce}(\mathrm{IV})$ in solution, and formate generated by hydrolysis of DMF. Dissolution of the $\mathrm{Ce}(\mathrm{IV})$-based MOF is driven by the decrease of $\mathrm{Ce}$ (IV) concentration in solution due to its reduction to $\mathrm{Ce}$ (III), which is continuously withdrawn from the reaction environment as $\mathrm{Ce}(\mathrm{III})$-formate. ${ }^{[12]}$

A recent article by some of us reported on the water-based synthesis of two perfluorinated Ce(IV)-based analogues of the already well investigated Zr(IV)-MOFs UiO-66 and MIL-140A (Figure 1). ${ }^{[13]}$ The pure MIL-140A phase, having formula [CeO(TFBDC) $\cdot \mathrm{H}_{2} \mathrm{O}$ ] (where TFBDC ${ }^{2-}$ is tetrafluoroterephthalate) and named F4_MIL$140 \mathrm{~A}(\mathrm{Ce})$, was obtained reacting stoichiometric amounts of cerium ammonium nitrate (CAN) and tetrafluoroterephthalic acid $\left(\mathrm{H}_{2}\right.$ TFBDC) in water at $110^{\circ} \mathrm{C}$ for $24 \mathrm{~h}$, whereas the pure UiO-66 phase, having formula $\left[\mathrm{Ce}_{6} \mathrm{O}_{4}(\mathrm{OH})_{4}(\mathrm{TFBDC})_{6}\right]$ and named F4_UiO-66(Ce), was obtained in the same conditions, with addition of 100 equivalents of acetic acid $(\mathrm{AcOH})$. In this case, no significant dissolution of the products was observed upon prolonged reaction time (up to $24 \mathrm{~h}$ ), most likely because of the different reaction medium than the classical DMF-based synthesis. Addition of $\mathrm{AcOH}$ in amounts smaller than 100 equivalents produced phase mixtures with varying ratios between the two phases. The coexistence of the two crystalline phases had also been previously observed in the $\mathrm{Zr}$-terephthalate system in DMF, in which UiO-66(Zr) is assumed to be the kinetic phase and MIL-140A(Zr) the thermodynamic phase, based on the observation that the former is preferentially obtained at lower temperatures than the latter, starting from reaction mixtures having the same composition. ${ }^{[14]}$ In our original report on $\mathrm{Ce}(\mathrm{IV})$-based perfluorinated MOFs, ${ }^{[13]}$ we speculated that in the Ce-TFBDC system in water the situation could be inverted, i.e. F4_UiO-66(Ce) is the thermodynamic phase and F4_MIL-140A(Ce) is the kinetic one, on the basis that a large amount of $\mathrm{AcOH}$ modulator was needed to obtain F4_UiO-66(Ce) in pure form in otherwise identical reaction conditions. In the case of polymorphs based on the same metal cluster, modulators have been seen to promote formation of thermodynamic products. ${ }^{[15]}$ However, UiO-66 is based on hexanuclear inorganic clusters, whereas MIL-140 contains infinite inorganic chains. It was recently shown that addition of 10 equivalents of $\mathrm{ACOH}$ to a DMF-based reaction mixture otherwise affording pure MIL-140A(Zr), a mixed MIL140A/UiO-66 product is formed. ${ }^{[16]}$ Further increasing the amount of acetic acid up to 30 equivalents appears to increase the MIL-140/UiO-66 ratio. These results may suggest that, at least in DMF solvent, the coordination modulator can in fact favour the thermodynamic product also in the MIL-140/UiO-66 system. 

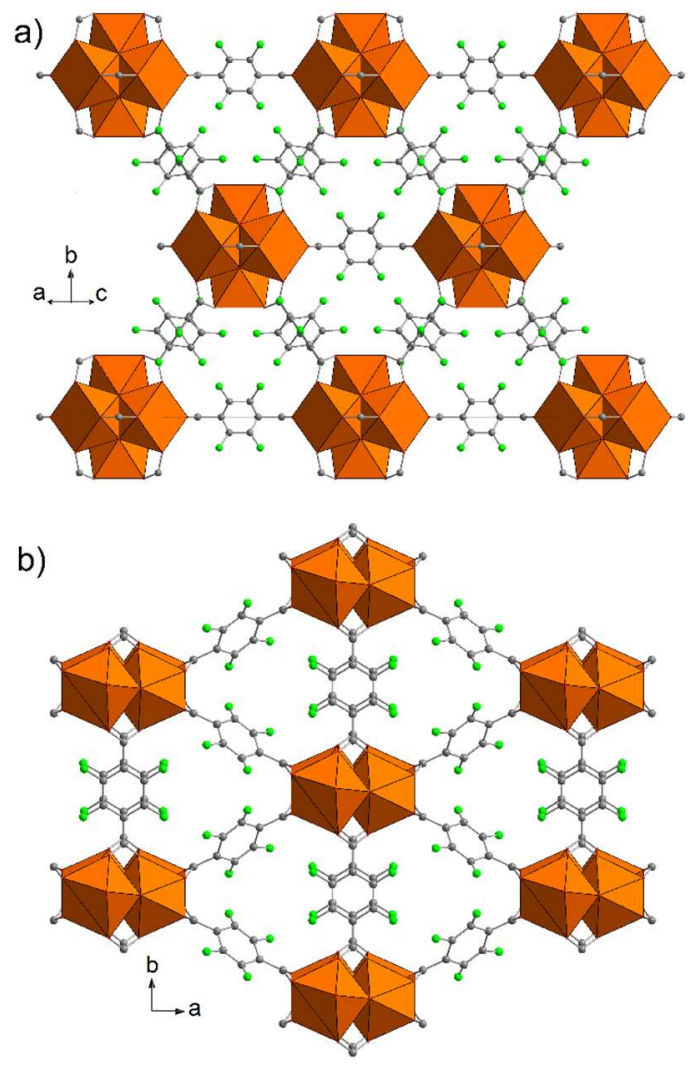

Figure 1. Crystal structures of F4_UiO-66(Ce) (a) and F4_MIL-140A(Ce) (b). Colour code: Ce, orange; F, green; O, red, C, grey. Reprinted with permission from ref [12]. Copyright 2019 American Chemical Society.

We therefore set out to employ in situ synchrotron PXRD to further understand how various reaction parameters influence the crystallisation process, both in terms of kinetics and thermodynamics, and to identify the range of parameters affording the two different F4_MIL-140A(Ce) and F4_UiO-66(Ce) single phases. In addition, since the MIL-140A analogue displayed promising $\mathrm{CO}_{2}$ capture properties - thanks to its non-hysteretic S-shaped isotherm and high $\mathrm{CO}_{2} / \mathrm{N}_{2}$ selectivity - we aimed at applying the knowledge deriving from the in situ investigation to identify an optimised synthesis protocol for the prospective production of this MOF on a multigram scale.

\section{Experimental Section}

\section{Materials}

Cerium(IV) ammonium nitrate (16774-21-3, 99\%, $\mathrm{Ce}\left(\mathrm{NH}_{4}\right)_{2}\left(\mathrm{NO}_{3}\right)_{6}$, Sigma-Merck), cerium(IV) sulfate (10294-425, 98\%, $\mathrm{Ce}\left(\mathrm{SO}_{4}\right)_{2} \cdot 4 \mathrm{H}_{2} \mathrm{O}$, Sigma-Merck), glacial acetic acid (64-19-7, 99\%, $\mathrm{CH}_{3} \mathrm{COOH}$ Sigma-Merck), nitric acid (7697-37-2, 68\%, $\mathrm{HNO}_{3}, \mathrm{VWR}$ ), sulfuric acid (7664-93-9, 95-97\%, $\mathrm{H}_{2} \mathrm{SO}_{4}$, Honeywell-Fluka), tetrafluoroterephthalic acid $\left(652-36-8,97 \%, \mathrm{C}_{8} \mathrm{H}_{2} \mathrm{~F}_{4} \mathrm{O}_{4}\right.$, Fluorochem). All reagents were used as received, with no further purification.

\section{In situ experiments}

The in situ study described hereafter took place at beamline P23 (DESY, Hamburg) and was carried out with the recently developed SynRAC (Synchrotron-based Reaction cell for the Analysis of Chemical reactions) unit. ${ }^{[17]}$ The SynRAC unit was specifically developed for in situ monitoring of solvothermal reactions using synchrotron X-raybased techniques. Figure S2 shows the unit in detail. The unit was designed to be simple, while incorporating a number of features that make it very versatile in its application. The reaction vessel, a simple $11 \mathrm{~mL}$ Pyrex reaction vial, is accommodated inside an aluminium casing and is surrounded by a heating mantle made from copper-galvanised heating wires which allows for fine control of the temperature up to $180{ }^{\circ} \mathrm{C}$. An electromagnetic stirrer is also incorporated for sample agitation up to $1200 \mathrm{rpm}$. 
We investigated two different systems: the first system involved only $\mathrm{HNO}_{3}$ as a protonation modulator and the effect of two parameters (temperature, amount of $\mathrm{HNO}_{3}$ ) was evaluated; the second system involved the combined use of $\mathrm{HNO}_{3}$ and $\mathrm{AcOH}$ as protonation and coordination modulators, respectively, and the effect of three parameters was evaluated (temperature, amount of $\mathrm{HNO}_{3}$, amount of $\mathrm{AcOH}$ ). For each reaction, we first dissolved $\mathrm{H}_{2}$ TFBDC in water, $\mathrm{HNO}_{3}$ and - where applicable - $\mathrm{AcOH}$, before heating the solution to the appropriate temperature under stirring inside the reaction chamber. The volume of this solution was $3.2 \mathrm{~mL}$ and the concentration of $\mathrm{H}_{2}$ TFBDC was $0.0625 \mathrm{~mol} \mathrm{~L}^{-1}$. On reaching the appropriate temperature, $0.8 \mathrm{~mL}$ of a $0.25 \mathrm{~mol} \mathrm{~L}^{-}$ ${ }^{1}$ solution of cerium ammonium nitrate (CAN) in water was injected, thus starting the reaction. Initially, there is a small drop in the temperature on addition of CAN, but this returns to the specified temperature within 1 minute. The final concentration of $\mathrm{H}_{2}$ TFBDC and CAN in the reaction mixture was $0.05 \mathrm{~mol} \mathrm{~L}^{-1}$.

XRD patterns were collected using $18.7 \mathrm{keV}(0.663 \AA$ A) radiation. The beamline was equipped with a X-Spectrum Lambda 750K 2D detector with GaAs sensor. The detector has an area of $512 \times 1528$ pixels ${ }^{2}$, with each pixel having size $55 \times 55 \mu \mathrm{m}$. The detector was positioned at $4^{\circ} 2 \theta$ from the incident beam direction, allowing us to span the angular region comprised between 0.47 and $7.37^{\circ} 2 \theta$. The acquisition time for each PXRD pattern was $2 \mathrm{~s}$, with an unavoidable delay time between successive measurements of $0.43 \mathrm{~s}$, hence providing a $2.43 \mathrm{~s}$ time resolution.

In order to extract any meaningful information from the raw data obtained from the in situ experiments, we used the DAWN software, an open source software funded primarily by Diamond Light Source, used for the visualisation and processing of scientific data, including XRD-type data. ${ }^{[18]}$ Full details about the data extraction procedure are provided in the SI (see the "Data processing using DAWN" section, Figures S2-4).

\section{Kinetic analysis}

The Gualtieri model was employed to extract kinetic information about the crystallisation process from the plots of the extent of crystallisation as a function of time. ${ }^{[1 b, 2 c, 19]}$ The Gualtieri model is a physically derived model which stemmed from experimental studies of the hydrothermal crystallisation of zeolites. ${ }^{[19]}$ This makes it an attractive model for crystallisation of MOFs under hydro- or solvothermal conditions. The reason this model is preferred over most others (e.g. Avrami-Erofeev) ${ }^{[1 \mathrm{~b}]}$ stems from two main factors. Firstly, the model is one based somewhat in reality on the way it was developed, i.e. looking at solvent-mediated reactions of solid reagents. Secondly, crystallisation is separated into two functions: nucleation and growth. This adds a new dimension to the understanding of the crystallisation process that cannot be obtained by other models.

The Gualtieri equation is the following:

$$
\alpha=\frac{1}{1+\exp \left\{-\left(\frac{t-a}{b}\right)\right\}} \cdot\left\{1-\exp \left[-\left(k_{g} t\right)^{n}\right]\right\}
$$

Where $\alpha$ is the extent of crystallisation as a function of time, $t . n$ represents the dimensionality of crystal growth, and is thus fixed depending on the type of crystals the material undergoing analysis forms. ${ }^{[2 c, 2 d, 19-20]}$ In our case, we are looking at F4_MIL-140A(Ce) and F4_UiO-66(Ce), the former of which forms rod-like crystals $(n=1)$, and the latter forms crystals with octahedral geometry $(n=3)$, as evidenced by SEM micrographs (Figure 2 ). The rate constant for crystal growth is represented by $k_{g}$. The constant $a$ is the reciprocal of the nucleation rate:

$$
k_{\mathrm{N}}=1 / a
$$

The constant $b$ describes the variance of the nucleation probability distribution. Using $a$ and $b$, the probability of nucleation, $P_{\mathrm{N}}$, can be calculated as follows:

$$
P_{\mathrm{N}}=\exp \left\{-\frac{(t-a)^{2}}{2 b^{2}}\right\}
$$

The Gualtieri equation was implemented in OriginPro as a user-defined non-linear equation and fittings of the experimental $\alpha$ vs $t$ plots were performed leaving $a, b$ and $k_{\mathrm{g}}$ free to refine and fixing $n$ to 1 for the F4_MIL$140 \mathrm{~A}(\mathrm{Ce})$ phase and 3 for the F4_UiO-66(Ce) phase. 

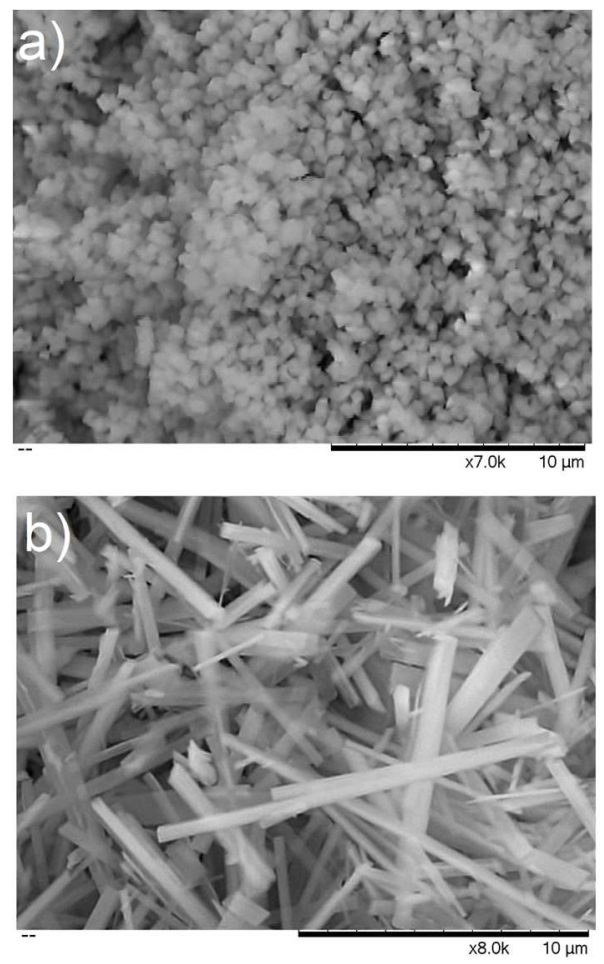

Figure 2. SEM micrographs of phase pure F4_UiO-66(Ce) (a) and F4_MIL-140A(Ce) (b), showing the typical octahedral morphology for the former and a needle-like morphology for the latter.

\section{Optimised synthesis of F4_MIL-140A(Ce)}

$\mathrm{H}_{2}$ TFBDC (179 mg, $0.75 \mathrm{mmol}$ ) is dissolved in water $\left(10.5 \mathrm{~mL}\right.$ ) and $16 \mathrm{~mol} \mathrm{~L}^{-1} \mathrm{HNO}_{3}(1.5 \mathrm{~mL}, 24 \mathrm{mmol})$ in a $20 \mathrm{~mL}$ scintillation vial, which is kept at $60^{\circ} \mathrm{C}$ under stirring either in an aluminium heating block or in an oil bath. After 10 minutes, a solution of CAN ( $411 \mathrm{mg}, 0.75 \mathrm{mmol})$ in water $(3 \mathrm{~mL})$ is added in the vial and the mixture is left to react for $1 \mathrm{~h}$. At the end of the reaction, the yellow solid is centrifuged, washed twice with water (15 $\mathrm{mL}$ each time) and finally washed with acetone $(15 \mathrm{~mL})$. The solid is dried in an oven at $80^{\circ} \mathrm{C}$. Yield: $177 \mathrm{mg}(60 \%)$.

\section{Ex situ analysis}

PXRD patterns were collected in the 4-30 $2 \theta$ range with a Bruker D8 Avance diffractometer working in reflection geometry and equipped with a LYNXEYE XE detector, using the $\mathrm{Cu}$ Ka radiation. The X-ray tube was operated at $40 \mathrm{kV}$ and $40 \mathrm{~mA}$.

Scanning electron microscopy (SEM) micrographs were collected using a Hitachi TM3030Plus Tabletop Microscope using an acceleration voltage of $5 \mathrm{kV}$. The samples were deposited on conductive copper tape on an aluminium support, without any sputtering process.

$\mathrm{N}_{2}$ sorption analysis at $77 \mathrm{~K}$ was performed on a Micromeritics ASAP 2020 gas sorption analyser. The sample (about $50 \mathrm{mg}$ ) was activated for $16 \mathrm{~h}$ at $120^{\circ} \mathrm{C}$ under dynamic vacuum prior to analysis. The BET equation was fitted in the 0.007-0.07 P/Po range.

High pressure $\mathrm{CO}_{2}$ adsorption isotherms up to 5 bar were measured with a Quantachrome iSorb High Pressure Gas Analyser at $25,40,55$ and $70^{\circ} \mathrm{C}$. About $200 \mathrm{mg}$ of sample was used for the adsorption studies. The sample was degassed at $120^{\circ} \mathrm{C}$ under dynamic vacuum for $12 \mathrm{~h}$ prior to analysis and at $120{ }^{\circ} \mathrm{C}$ for $1 \mathrm{~h}$ in between subsequent measurements.

\section{Results and Discussion}

\section{Preliminary synthetic screening}

We started our investigation performing preliminary in situ PXRD experiments employing analogous conditions to those reported in the original paper describing the synthesis of F4_MIL-140A(Ce) and F4_UiO-66(Ce): for the 
former, we dissolved $0.21 \mathrm{mmol} \mathrm{H}_{2}$ TFBDC in $2 \mathrm{~mL}$ of water, heated the mixture up to $110{ }^{\circ} \mathrm{C}$, then added 0.21 mmol of CAN dissolved in $0.4 \mathrm{~mL}$ of water; for the latter, $1 \mathrm{~mL}$ water was replaced by $1 \mathrm{~mL} \mathrm{AcOH}$, keeping the same total volume. These experiments clearly showed that crystallisation was complete within a few seconds from introduction of $\mathrm{Ce}(\mathrm{IV})$ in the reaction vessel, too fast to be followed with satisfactory time resolution (Figure S5).

This pushed us to investigate options to gain control of the crystallisation kinetics, with the primary aim of slowing the process down and make it occur within a timeframe in the range of a few minutes, compatible with in situ monitoring using XRD. In order to ensure full reproducibility, the experiments discussed in this section were performed using the same types of reaction vessels and stir bars to be used for the in situ study. We chose to test $\mathrm{HNO}_{3}$ as a protonation modulator, under the hypothesis that, being a strong acid, it should inhibit deprotonation of $\mathrm{H}_{2}$ TFBDC, making it less available for coordination to the metal, while releasing nitrate ions in solution, i.e. the same anion present in the metal precursor CAN, thus not introducing a new species in the reaction environment. Addition of $1 \mathrm{~mL}$ of $16 \mathrm{~mol} \mathrm{~L}^{-1} \mathrm{HNO}_{3}$ (64 equivalents) to an equimolar mixture of $\mathrm{H}_{2} \mathrm{TFBDC}$ and CAN ( $0.25 \mathrm{mmol}$ each) in $4 \mathrm{~mL}$ of $\mathrm{H}_{2} \mathrm{O}$ completely prevented crystallisation at $32{ }^{\circ} \mathrm{C}$, suggesting that the desired modulating effect was taking place. Notably, even upon heating the solution to $80{ }^{\circ} \mathrm{C}$ no solid formed, whereas the solution turned from dark yellow to non-coloured within $2 \mathrm{~h}$. This suggests that, when crystallisation is inhibited, reduction of $\mathrm{Ce}$ (IV) to $\mathrm{Ce}$ (III), likely accompanied by water oxidation, becomes the dominant process occurring in the reaction mixture.

These observations were the starting point for an extensive synthetic screening aimed at determining a range of conditions to be investigated in situ. Full results, including PXRD patterns of the products, are reported in the SI (Tables S1-2 and Figures S6-10). We list herein only the most important observations made during this screening, which guided the choice of the range of parameters to investigate during the in situ PXRD study.

- $\quad$ F4_MIL-140A(Ce) was the sole crystalline phase obtained when only $\mathrm{HNO}_{3}$ was added to the reaction mixture as a modulator.

- Keeping the concentration of $\mathrm{H}_{2}$ TFBDC and CAN fixed at 0.05 mol L-1 and varying the amount of $\mathrm{HNO}_{3}$ added, we found that, above 32 equivalents of $\mathrm{HNO}_{3}$, precipitation of the MOF was inhibited and the solution lost colour within $2 \mathrm{~h}$ of heating at both 80 and $100^{\circ} \mathrm{C}$, indicating reduction of $\mathrm{Ce}(\mathrm{IV})$ to $\mathrm{Ce}(\mathrm{III})$.

- Keeping the amount of $\mathrm{HNO}_{3}$ fixed at 32 equivalents and varying the temperature between 50 and 80 ${ }^{\circ} \mathrm{C}$, we observed that appearance of the solid occurred progressively earlier as the temperature increased, suggesting that temperature has a significant effect on the crystallisation kinetics.

- $\quad$ The F4_UiO-66(Ce) phase was only accessible when $\mathrm{AcOH}$ was also present in the reaction environment and could be obtained in pure form in the presence of 6.4 equivalents of $\mathrm{HNO}_{3}$ and at least 147 equivalents of $\mathrm{AcOH}$.

- Increasing the amount of $\mathrm{HNO}_{3}$ to 16 equivalents or lowering the $\mathrm{AcOH}$ amount to 96 equivalents or less yielded mixed-phase products, with varying phase ratios depending on the $\mathrm{HNO}_{3} / \mathrm{AcOH}$ ratio.

- $\quad$ No solid formation was observed when reactions were carried out in the presence of 32 equivalents of $\mathrm{HNO}_{3}$ and 98 equivalents of $\mathrm{AcOH}$ at $70{ }^{\circ} \mathrm{C}$, as well as in the presence of 16 equivalents of $\mathrm{HNO}_{3}$ and 196 equivalents of $\mathrm{AcOH}$ at $70^{\circ} \mathrm{C}$.

Based on these observations, the parameter space displayed in Figure 3 was investigated in the in situ PXRD study.

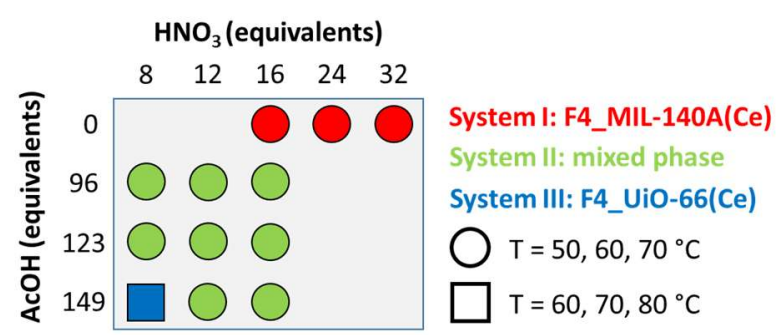

Figure 3. Three systems were chosen to investigate the parameter space for the synthesis of F4_MIL-140A(Ce) and F4_UiO66(Ce). One equivalent corresponds to $0.25 \mathrm{mmol}$. The molar ratio $\mathrm{Ce}^{4+}$ to $\mathrm{H}_{2}$ TFBDC is fixed at a value of 1 to 1 . 


\section{In situ PXRD study}

\section{System I: phase pure F4_MIL-140A(Ce)}

In the exploration of this system, only $\mathrm{HNO}_{3}$ was used as a modulator (Figure 3). We kept the concentration of CAN and $\mathrm{H}_{2}$ TFBDC fixed at $0.05 \mathrm{~mol} \mathrm{~L}^{-1}$ and looked independently at two parameters - temperature and amount of $\mathrm{HNO}_{3}$ - in order to assess their effect on the rates of nucleation and crystal growth. The product we observed for this system was always a single-phase F4_MIL-140A(Ce).

\section{Effect of temperature}

In assessing the effect of temperature, we varied between 50,60 , and $70{ }^{\circ} \mathrm{C}$, while keeping the amount of $\mathrm{HNO}_{3}$ fixed at 32 equivalents. We observed that the increase in temperature greatly amplified the rate of crystallisation (Figure 4). The probability of nucleation $\left(P_{\mathrm{N}}\right)$, displayed in the form of the dashed lines in Figure 4 , peaks in correspondence of progressively lower $\alpha$ values as the temperature increases $\left(\alpha=0.50\right.$ when $P_{\mathrm{N}}$ peaks at $50{ }^{\circ} \mathrm{C}$, $\alpha=0.25$ when $P_{\mathrm{N}}$ peaks at $60^{\circ} \mathrm{C}, \alpha=0.12$ when $P_{\mathrm{N}}$ peaks at $70{ }^{\circ} \mathrm{C}$ ), suggesting that nucleation is more sensitive than crystal growth to changes in temperature. Fitting the Gualtieri equation to each set of data (Figure S11), the results reported in Table 1 and Table S3 were extracted. These results show in fact that the nucleation rate at $70{ }^{\circ} \mathrm{C}$ is 33 times greater than at $50^{\circ} \mathrm{C}$, whereas the crystal growth rate only increases by a factor of 4 in the same range. Nucleation is rate determining at $50{ }^{\circ} \mathrm{C}\left(k_{\mathrm{N}}<k_{\mathrm{g}}\right)$, but already at $60{ }^{\circ} \mathrm{C}$ the situation is inverted, and crystal growth becomes rate determining. The $k_{\mathrm{N}} / k_{\mathrm{g}}$ ratio increases from 0.46 to 1.66 to 3.66 at 50,60 and 70 ${ }^{\circ} \mathrm{C}$, respectively (Table 1 ).

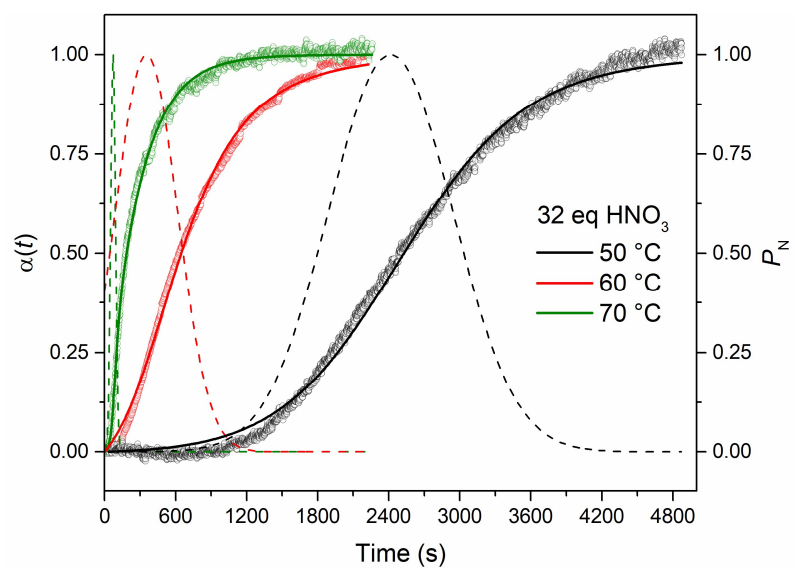

Figure 4. Plot of extent of crystallization ( $\alpha$, empty circles), Gualtieri fitting (solid lines) and probability of nucleation $\left(P_{\mathrm{N}}\right.$, dashed lines) as a function of time for experiments performed at $50^{\circ} \mathrm{C}$ (black), $60{ }^{\circ} \mathrm{C}$ (red) and $70{ }^{\circ} \mathrm{C}$ (green) in the presence of 32 equivalents of $\mathrm{HNO}_{3}$, leading to the formation of phase pure F4_MIL-140A(Ce).

Table 1. Rate constants obtained for F4_MIL-140A(Ce) crystallisation in the presence of 32 equivalents $\left(0.4 \mathrm{~mL}, 1.6 \mathrm{~mol} \mathrm{L^{-1 }}\right)$ of $\mathrm{HNO}_{3}$ at 50,60 , and $70^{\circ} \mathrm{C}$. Full Gualtieri fitting parameters with errors are given in Table S3.

\begin{tabular}{cccccc}
\hline $\begin{array}{c}\text { Temp } \\
\left({ }^{\circ} \mathrm{C}\right)\end{array}$ & $\begin{array}{c}k_{\mathrm{g}} \\
\left(\mathbf{s}^{-1}\right)\end{array}$ & $\begin{array}{c}\text { Relative } \\
\boldsymbol{k}_{\mathrm{g}}\end{array}$ & $\begin{array}{c}\boldsymbol{k}_{\mathrm{N}} \\
\left(\mathbf{s}^{-1}\right)\end{array}$ & $\begin{array}{c}\text { Relative } \\
\boldsymbol{k}_{\mathrm{N}}\end{array}$ & $\begin{array}{c}\text { Ratio } \\
\boldsymbol{k}_{\mathrm{N}} / \mathbf{k}_{\mathrm{g}}\end{array}$ \\
\hline $\mathbf{7 0}$ & 0.00376 & 4.0 & 0.0139 & 33.5 & 3.66 \\
$\mathbf{6 0}$ & 0.00168 & 1.8 & 0.00283 & 6.8 & 1.66 \\
$\mathbf{5 0}$ & 0.00093 & 1.0 & 0.000414 & 1.0 & 0.46 \\
\hline
\end{tabular}

By plotting the natural logarithm of $k_{\mathrm{N}}$ and $k_{\mathrm{g}}$ versus $1 / \mathrm{T}$, the activation energy for nucleation and crystal growth can be extracted by simple linear fitting, according to the logarithmic form of the Arrhenius equation. Figure 5 shows the fits for both nucleation and crystal growth. The two curves cross over at a value of $1 / T$ corresponding to about $57^{\circ} \mathrm{C}$ : below this temperature nucleation is the rate determining step, whereas above this temperature crystal growth becomes rate determining. The extracted activation energies are $154 \pm 4 \mathrm{~kJ} \mathrm{~mol}^{-1}$ for nucleation 
$\left(E_{a, \mathrm{~N}}\right)$ and $63 \pm 6 \mathrm{~kJ} \mathrm{~mol}^{-1}$ for crystal growth $\left(E_{\mathrm{a}, \mathrm{g}}\right)$. These values are in line with those of MOFs such as MOF-14 $\left(E_{a, N}=114 \mathrm{~kJ} \mathrm{~mol}^{-1}, E_{\mathrm{a}, \mathrm{g}}=83 \mathrm{~kJ} \mathrm{~mol}^{-1}\right)^{[2 \mathrm{e}]}$ and Mn-MIL-100 $\left(E_{\mathrm{a}, \mathrm{N}}=127 \mathrm{~kJ} \mathrm{~mol}^{-1}, E_{\mathrm{a}, \mathrm{g}}=99 \mathrm{~kJ} \mathrm{~mol}^{-1}\right)^{[21]}$, whereby nucleation has a considerably higher activation energy than growth. However, neither MOF-14 nor Mn-MIL-140 show any crossing of the curves in the range of temperatures investigated, which means that nucleation is always the rate determining step. There are also examples of MOFs where the activation energies for nucleation and growth are relatively similar, which include ZIF-8 $\left(E_{\mathrm{a}, \mathrm{N}}=69 \mathrm{~kJ} \mathrm{~mol}^{-1}, E_{\mathrm{a}, \mathrm{g}}=72 \mathrm{~kJ} \mathrm{~mol}^{-1}\right)^{[2 \mathrm{~d}]}$, HKUST-1 $\left(E_{\mathrm{a}, \mathrm{N}}=72\right.$ $\left.\mathrm{kJ} \mathrm{mol}{ }^{-1}, E_{a, g}=64 \mathrm{~kJ} \mathrm{~mol}^{-1}\right)^{[2 \mathrm{e}]}$, and Zr-fumarate $\left(E_{\mathrm{a}, \mathrm{N}}=71 \mathrm{~kJ} \mathrm{~mol}^{-1}, E_{\mathrm{a}, \mathrm{g}}=66 \mathrm{~kJ} \mathrm{~mol}^{-1}\right) \cdot{ }^{[2 \mathrm{c}]}$ Given the very similar slopes of the curves, the rate determining step for these systems is always the same (i.e. nucleation in the case of both HKUST-1 and Zr-Fumarate, growth for ZIF-8) over a wide range of temperatures. Rather interestingly, the activation energies for $\mathrm{Zr}$-UiO-66 seem to be much lower with growth as the limiting factor $\left(E_{\mathrm{a}, \mathrm{N}}=11-39 \mathrm{~kJ}\right.$ $\mathrm{mol}^{-1}, E_{\mathrm{a}, \mathrm{g}}=19-46 \mathrm{~kJ} \mathrm{~mol}^{-1}$ ), depending on both the amount of $\mathrm{HCl}$ present and the source of $\mathrm{Zr}$ used..$^{[22]}$

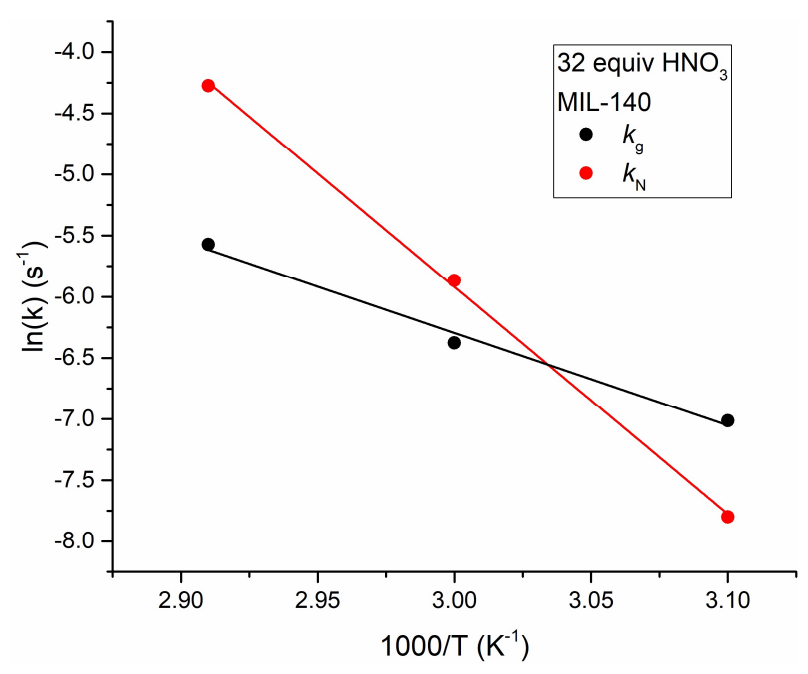

Figure 5. Plot of $\ln \left(k_{\mathrm{N}}\right)$ (red circles) and $\ln \left(k_{\mathrm{g}}\right)$ (black circles) versus $1 / \mathrm{T}$ for experiments performed at 50,60 and $70{ }^{\circ} \mathrm{C}$ in the presence of 32 equivalents of $\mathrm{HNO}_{3}$, leading to phase pure F4_MIL-140A(Ce). The red line and the black line are the linear Arrhenius fittings for nucleation and crystal growth, respectively.

\section{Effect of $\mathrm{HNO}_{3}$ amount}

The second parameter we explored in this system was the amount of $\mathrm{HNO}_{3}$, whose number of equivalents was varied in the order of 32-24-16, keeping the temperature at $60^{\circ} \mathrm{C}$ throughout. We observed that decreasing the amount of $\mathrm{HNO}_{3}$ led to a large increase in the rate of crystallisation (Figure 6). While $P_{\mathrm{N}}$ for 32 equivalents of $\mathrm{HNO}_{3}$ peaks after $350 \mathrm{~s}$, for 24 and 16 equivalents this drops to 18 and $5 \mathrm{~s}$, respectively (Figure 6). However, $P_{\mathrm{N}}$ peaks at about $0.25 \alpha$ in all cases, suggesting that nucleation and crystal growth rates evolve in a similar manner. As a matter of fact, Gualtieri fitting showed that the effect of $\mathrm{HNO}_{3}$ on both the rate of nucleation and growth was relatively similar, with both being about 68 times greater at 16 equivalents of $\mathrm{HNO}_{3}$ than they were at 32 equivalents (Table 2, Table S4, Figure S12). Crystal growth is rate determining at each amount of $\mathrm{HNO}_{3}$ added. The $k_{\mathrm{N}} / \mathrm{k}_{\mathrm{g}}$ ratio changes from 1.66 (32 equivalents) to 2.10 (24 equivalents) to 1.69 (16 equivalents), confirming that $\mathrm{HNO}_{3}$ affects nucleation and growth basically to the same extent (Table 2). This is also evident from the plot of $\ln \left(k_{\mathrm{N}}\right)$ and $\ln \left(k_{\mathrm{g}}\right)$ versus the amount of $\mathrm{HNO}_{3}$ (Figure 7). 


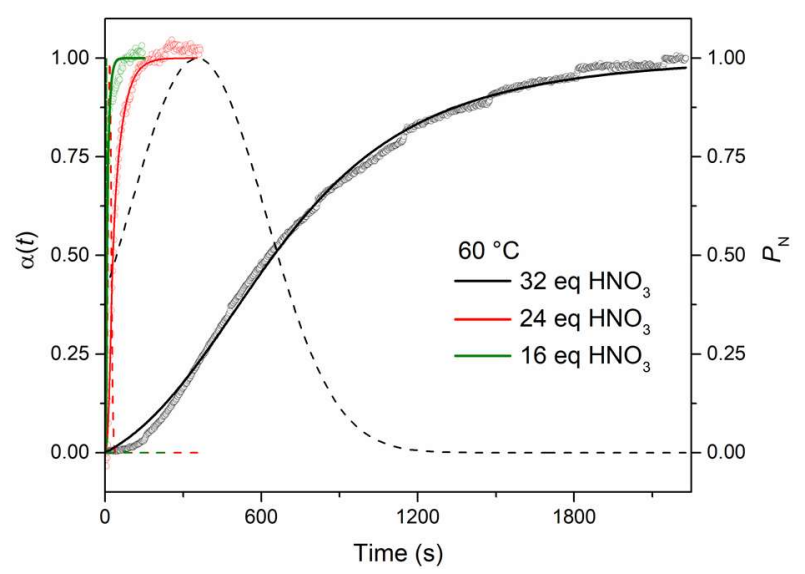

Figure 6. Plot of extent of crystallization ( $\alpha$, empty circles), Gualtieri fitting (solid lines) and probability of nucleation $\left(P_{N}\right.$, dashed lines) as a function of time for experiments performed at $60{ }^{\circ} \mathrm{C}$ using 32 (black), 24 (red) and 16 (green) equivalents of $\mathrm{HNO}_{3}$ for the F4_MIL-140A(Ce) phase.

Table 2. Rate constants obtained for F4_MIL-140A(Ce) crystallisation in the presence of 16 equivalents $\left(0.2 \mathrm{~mL}, 0.8 \mathrm{~mol} \mathrm{~L}^{-1}\right)$, 24 equivalents $\left(0.3 \mathrm{~mL}, 1.2 \mathrm{~mol} \mathrm{~L}^{-1}\right)$, and 32 equivalents $\left(0.4 \mathrm{~mL}, 1.6 \mathrm{~mol} \mathrm{~L}^{-1}\right)$ of $\mathrm{HNO}_{3}$ at $60{ }^{\circ} \mathrm{C}$. Full Gualtieri fitting parameters with errors are given in Table S4.

\begin{tabular}{cccccc}
\hline $\begin{array}{c}\mathrm{HNO}_{3} \\
(\mathbf{e q})\end{array}$ & $\begin{array}{c}\boldsymbol{k}_{\mathrm{g}} \\
\left(\mathbf{s}^{-1}\right)\end{array}$ & $\begin{array}{c}\text { Relative } \\
\boldsymbol{k}_{\mathrm{g}}\end{array}$ & $\begin{array}{c}\boldsymbol{k}_{\mathrm{N}} \\
\left(\mathbf{s}^{-1}\right)\end{array}$ & $\begin{array}{c}\text { Relative } \\
\boldsymbol{k}_{\mathrm{N}}\end{array}$ & $\begin{array}{c}\text { Ratio } \\
\boldsymbol{k}_{\mathrm{N}} / \boldsymbol{k}_{\mathrm{g}}\end{array}$ \\
\hline $\mathbf{1 6}$ & 0.114 & 67.9 & 0.19 & 67.9 & 1.69 \\
$\mathbf{2 4}$ & 0.0267 & 15.9 & 0.056 & 19.8 & 2.10 \\
$\mathbf{3 2}$ & 0.00168 & 1.0 & 0.00283 & 1.0 & 1.66 \\
\hline
\end{tabular}

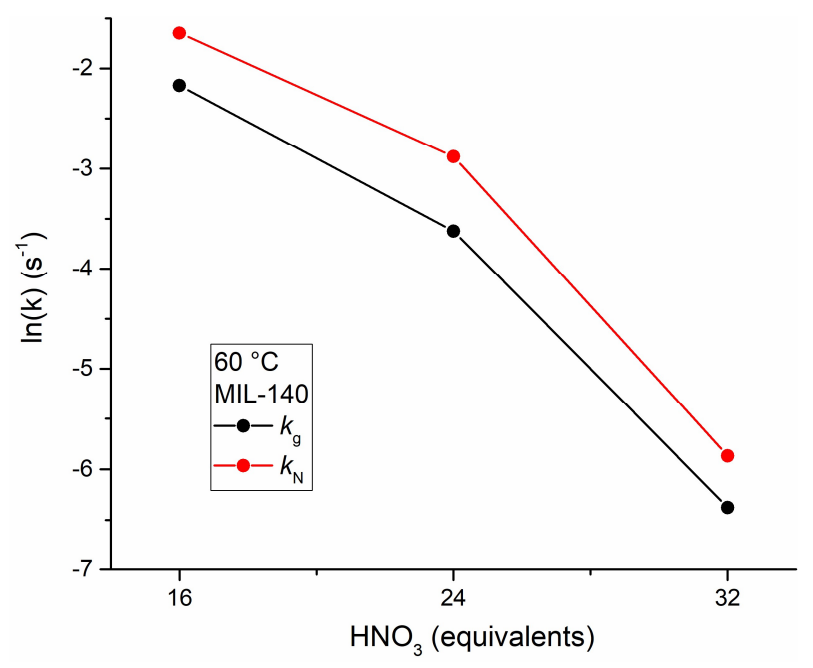

Figure 7. Plot of $\ln \left(k_{\mathrm{N}}\right)$ (red circles) and $\ln \left(k_{\mathrm{g}}\right.$ ) (black circles) versus the amount of $\mathrm{HNO}_{3}$ for experiments performed at $60^{\circ} \mathrm{C}$ in the presence of 16,24 and 32 equivalents of $\mathrm{HNO}_{3}$. Lines are a guide for the eye.

\section{System II: mixed phase F4_MIL-140A(Ce)/F4_UiO-66(Ce)}

The second system we explored involved the addition of $\mathrm{AcOH}$, which was used as a coordination modulator, besides $\mathrm{HNO}_{3}$. In the exploration of this system, we kept the concentration of CAN and $\mathrm{H}_{2}$ TFBDC fixed at 0.05 mol L-1 and looked independently at three parameters - temperature, amount of $\mathrm{HNO}_{3}$ and amount of $\mathrm{AcOH}$ - in order to assess their effect on the rates of nucleation and crystal growth of the two crystalline phases [F4_MIL140A(Ce) and F4_UiO-66(Ce)] formed during these experiments.

\section{Effect of temperature}


As with the first system, the first parameter we looked at was the effect of temperature on crystallisation, varying in the range 50,60 and $70^{\circ} \mathrm{C}$, while $\mathrm{HNO}_{3}$ and $\mathrm{AcOH}$ were kept fixed at 16 equivalents and 96 equivalents, respectively. Comparing the plots of $\alpha$ vs $t$ for the two phases at each temperature (Figure 8 ), it is evident that the F4_UiO-66(Ce) phase is formed first at each temperature. This provides experimental evidence that F4_UiO$66(\mathrm{Ce})$ is in fact the kinetically favoured phase, in line with what originally suggested for the Zr-terephthalate system in $\mathrm{DMF}^{[14]}$ when $\mathrm{AcOH}$ is present in the reaction environment for the Ce-TFBDC water-based synthesis. It is plausible that $\mathrm{AcOH}$ plays a role in promoting solution templation of discrete clusters, which are essential to enable the formation of the UiO-66 framework in aqueous environment through a ligand exchange process whereby TFBDC ${ }^{2-}$ replaces acetate. At both 50 and $60^{\circ} \mathrm{C}$ (Figures S13-14), we observe some drop in the intensity for the F4_UiO-66(Ce) phase upon prolonged reaction, which might suggest either some degradation or consumption of this phase due to interconversion into F4_MIL-140A(Ce). However, the drop in intensity of F4_UiO-66(Ce) starts after most of F4_MIL-140A(Ce) has already formed and, looking at the plots reporting absolute intensities for both experiments, it is clear that the small drop in intensity of F4_UiO-66(Ce) cannot account for the large increase seen in the intensity of F4_MIL-140A(Ce). This suggests that the two phases form independently and there is no significant interconversion between them. Rather, the two phases compete for reagents present in solution. Furthermore, the intensity of $\mathrm{F4}$ _UiO-66(Ce) plateaus after some time, suggesting that decomposition of this MOF does not continue until complete disappearance.

In the experiments performed at 50 and $60^{\circ} \mathrm{C}$ (Figure 8a-b), $P_{\mathrm{N}}$ for F4_MIL-140A(Ce) peaks when F4_UiO-66(Ce) is already completely crystallised. The experiment performed at $70^{\circ} \mathrm{C}$ (Figure $8 \mathrm{C}$ ) shows instead that the curve for F4_UiO-66(Ce) experiences a sudden change in slope after about $30 \mathrm{~s}$, when it has reached $\alpha$ of about 0.63 and its $P_{\mathrm{N}}$ is at zero, substantially decreasing its rate of crystal growth. Interestingly, this event occurs when the $P_{\mathrm{N}}$ for F4_MIL-140A(Ce) reaches its peak, suggesting that growth of F4_UiO-66(Ce) in these conditions could be inhibited by competition with F4_MIL-140A(Ce) for reagents. Looking at the evolution of the intensity ratio between the two phases at full crystallisation, we can have a proxy of the effect of a parameter on the relative yield of each phase. We used the intensity of the 110 reflection for the F4_MIL-140A(Ce) phase and the intensity of the 111 reflection for the $\mathrm{F4}$ _UiO-66(Ce) phase at full crystallisation to extract MIL/UiO ratios of 1.5 at $50{ }^{\circ} \mathrm{C}$, 1.3 at $60^{\circ} \mathrm{C}$ and 1.7 at $70{ }^{\circ} \mathrm{C}$, thus observing no obvious trend due to the temperature increase. It is to be noted, though, that the intensity ratio at $70^{\circ} \mathrm{C}$ is probably affected by the deceleration of $\mathrm{F} 4$ _UiO-66(Ce) crystallisation, which is likely to lead to a lower amount of this phase being formed at the end of the reaction than if there was no competition with F4_MIL-140A(Ce). 

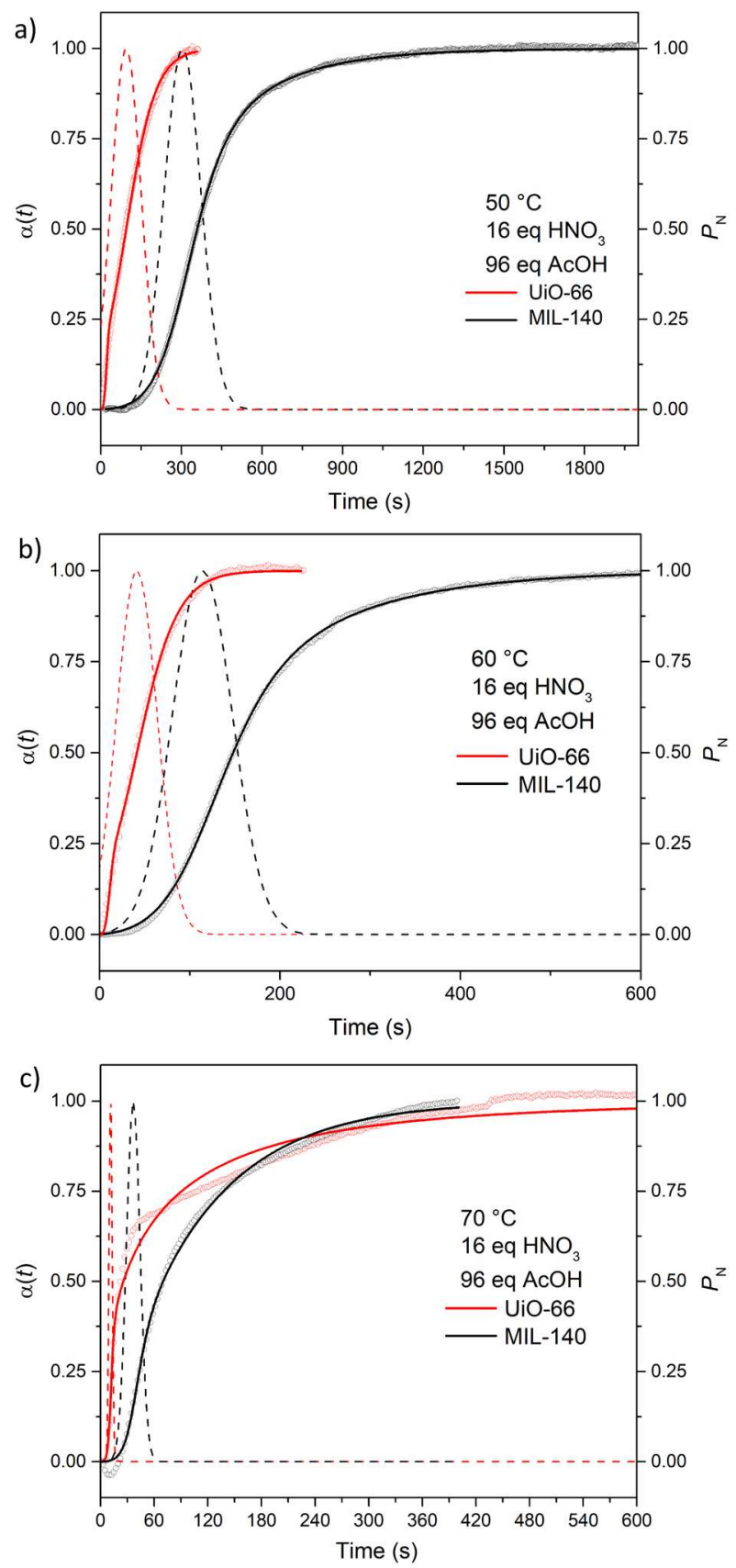

Figure 8. Plot of extent of crystallization ( $\alpha$, empty circles), Gualtieri fitting (solid lines) and probability of nucleation $\left(P_{\mathrm{N}}\right.$, dashed lines) as a function of time for experiments performed at $50{ }^{\circ} \mathrm{C}(\mathrm{a}), 60^{\circ} \mathrm{C}(\mathrm{b})$ and $70{ }^{\circ} \mathrm{C}$ (c) for the F4_UiO-66(Ce) (red) and F4_MIL-140A(Ce) (black) phases.

Focusing on the crystallisation kinetics of the F4_MIL-140A(Ce) phase, it is clear that temperature increases the rate of crystallisation (Figure 9a), and, as already observed in the absence of $\mathrm{AcOH}$, this is affected more by the rate of nucleation than growth (Table 3, Table S5, Figure S15). One thing that is also clear is that the addition of $\mathrm{AcOH}$ has dampened the $E_{\mathrm{a}, \mathrm{N}}$ when compared with the first system, with this one having a $k_{\mathrm{N}}$ ratio between 50 and $70{ }^{\circ} \mathrm{C}$ of $1: 8$, compared with $1: 34$ for the first system. The $k_{\mathrm{N}} / \mathrm{kg}_{\mathrm{g}}$ ratio changes from $0.92\left(50{ }^{\circ} \mathrm{C}\right)$ to $1.14(60$ ${ }^{\circ} \mathrm{C}$ ) to $2.73\left(70^{\circ} \mathrm{C}\right)$, suggesting that, also in this system, temperature accelerates nucleation preferentially over crystal growth, with the latter becoming increasingly rate determining as the temperature increases. This can also be seen from the plot of $P_{\mathrm{N}}$ (Figure 9a), which peaks in correspondence of progressively lower $\alpha$ as the temperature increases. Comparing the values of $k_{\mathrm{N}}$ and $k_{\mathrm{g}}$ for the experiments conducted at $60^{\circ} \mathrm{C}$ in the presence of 16 equivalents of $\mathrm{HNO}_{3}$, the effect of the addition of $\mathrm{AcOH}$ on nucleation and growth can be assessed. In the presence of 96 equivalents of $\mathrm{AcOH}, k_{\mathrm{N}}$ is $0.00877 \mathrm{~s}^{-1}$ and $k_{\mathrm{g}}$ is $0.00769 \mathrm{~s}^{-1}$ (Table 3), whereas in the absence of $\mathrm{AcOH} k_{\mathrm{N}}$ is $0.19231 \mathrm{~s}^{-1}$ and $k_{\mathrm{g}}$ is $0.1140 \mathrm{~s}^{-1}$ (Table 1), indicating that $\mathrm{AcOH}$ dampens both nucleation and crystal 
growth rates, but the first is more affected, decreasing by a factor of 22, versus growth, decreasing by a factor of 15 , when $\mathrm{AcOH}$ is added into the reaction environment.
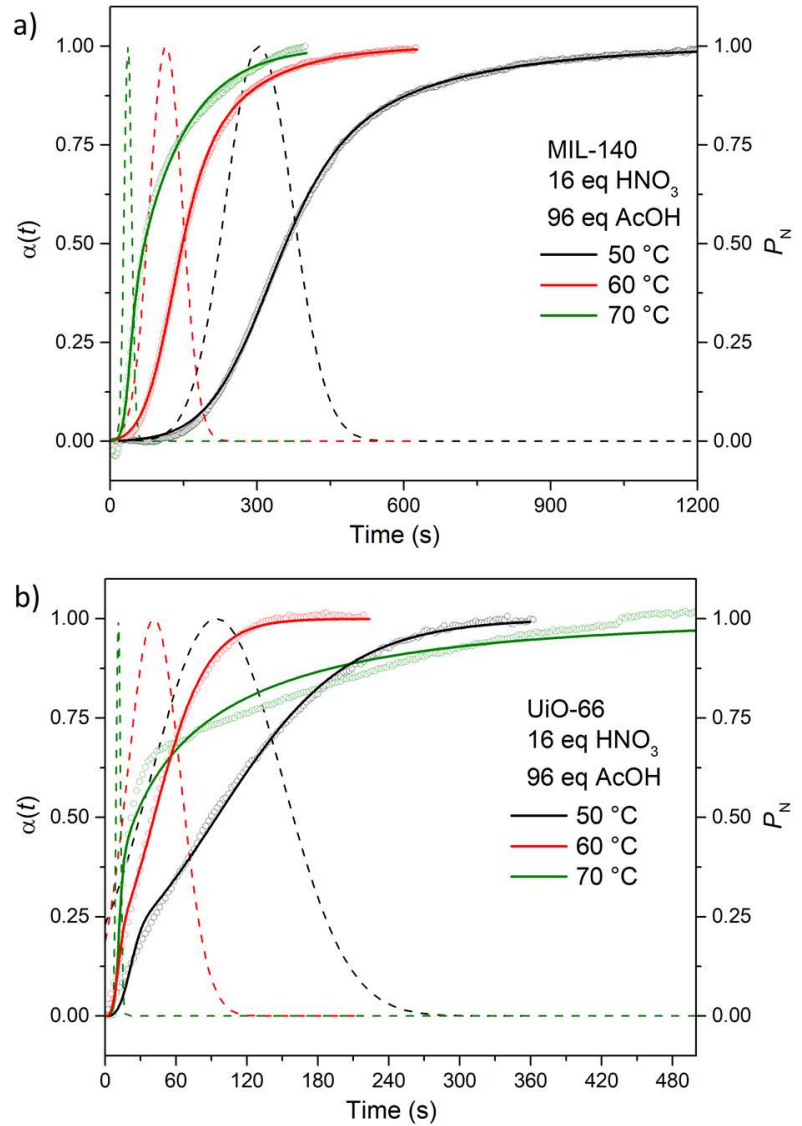

Figure 9. Plot of extent of crystallization ( $\alpha$, empty circles), Gualtieri fitting (solid lines) and probability of nucleation $\left(P_{\mathrm{N}}\right.$, dashed lines) as a function of time for experiments performed in the presence of 16 equivalents of $\mathrm{HNO}_{3}$ and 96 equivalents of $\mathrm{AcOH}$ at $50{ }^{\circ} \mathrm{C}$ (black), $60^{\circ} \mathrm{C}$ (red) and $70^{\circ} \mathrm{C}$ (green) for the F4_MIL-140A(Ce) phase (a) and the F4_UiO-66(Ce) phase (b).

Table 3. Rate constants obtained for F4_MIL-140A(Ce) and F4_UiO-66(Ce) in the presence of 16 equivalents $(0.2 \mathrm{~mL}, 0.8 \mathrm{~mol}$ $\left.\mathrm{L}^{-1}\right)$ of $\mathrm{HNO}_{3}$, and 96 equivalents $\left(1.1 \mathrm{~mL}, 4.8 \mathrm{~mol} \mathrm{~L}^{-1}\right)$ of $\mathrm{AcOH}$ at 50,60 , and $70^{\circ} \mathrm{C}$. Full Gualtieri fitting parameters with errors are given in Table $\mathrm{S} 5$.

\begin{tabular}{|c|c|c|c|c|c|c|}
\hline & $\begin{array}{c}\text { Temp } \\
\left({ }^{\circ} \mathrm{C}\right)\end{array}$ & $\begin{array}{c}k_{\mathrm{g}} \\
\left(\mathrm{s}^{-1}\right)\end{array}$ & $\begin{array}{c}\text { Relative } \\
k_{\mathrm{g}}\end{array}$ & $\begin{array}{c}k_{N} \\
\left(s^{-1}\right)\end{array}$ & $\begin{array}{c}\text { Relative } \\
k_{\mathrm{N}}\end{array}$ & $\begin{array}{l}\text { Ratio } \\
k_{\mathrm{N}} / k_{\mathrm{B}}\end{array}$ \\
\hline \multirow{3}{*}{$\begin{array}{l}\text { o } \\
\stackrel{\sigma}{\vec{J}} \\
\stackrel{\vec{J}}{\Sigma}\end{array}$} & 70 & 0.01008 & 2.8 & 0.0273 & 8.3 & 2.73 \\
\hline & 60 & 0.00769 & 2.1 & 0.00877 & 2.7 & 1.14 \\
\hline & 50 & 0.00359 & 1.0 & 0.003289 & 1.0 & 0.92 \\
\hline \multirow{3}{*}{$\begin{array}{l}\text { o } \\
\text { 1 } \\
\text { j. }\end{array}$} & $70 *$ & 0.0200 & $0.5^{* *}$ & 0.088 & 8.6 & 4.55 \\
\hline & 60 & 0.086 & 2.0 & 0.0242 & 2.3 & 0.28 \\
\hline & 50 & 0.044 & 1.0 & 0.01060 & 1.0 & 0.24 \\
\hline
\end{tabular}

${ }^{*} n$ had to be left free to refine to have a reasonable fitting, returning a value of $0.55 \pm 0.01 ; * *$ Value not reliable due to the change in rate during crystallisation

The F4_UiO-66(Ce) phase also shows a similar trend to F4_MIL-140A(Ce), in that the $k_{\mathrm{N}}$ ratio between 50 and 70 ${ }^{\circ} \mathrm{C}$ is $1: 9$, and again has a larger contribution to the overall rate of crystallisation than the rate of growth (Figure $9 \mathrm{~b}$, Table 3, Table S5). Both at 50 and $60{ }^{\circ} \mathrm{C}$, nucleation is rate determining. Due to the change in slope of the curve at $70{ }^{\circ} \mathrm{C}$, a reliable value for $k_{\mathrm{g}}$ at this temperature could not be extracted. The $k_{\mathrm{N}} / \mathrm{kg}_{\mathrm{g}}$ ratio changes from $0.24\left(50^{\circ} \mathrm{C}\right)$ to $0.28\left(60^{\circ} \mathrm{C}\right)$, suggesting that temperature accelerates nucleation and growth of F4_UiO-66(Ce) to the same extent (Table 3, Figure S16). The fitting of the Gualtieri model in this case seemed to present some 
issues, whereby the model does not reproduce the experimental data very well, especially in the initial stages of crystallisation. This can be identified in Figure S16, where the red line that represents the Gualtieri fit deviates from the experimental points at the start of the curve. A similar behaviour was reported when modelling the crystallisation kinetics of formate-modulated ZIF- $8{ }^{[2 \mathrm{[d]}]}$ We believe that the most likely reason for this effect is the lack of induction time for F4_UiO-66(Ce), whose crystallisation starts immediately as $\mathrm{Ce}(\mathrm{IV})$ is introduced in the system. Nonetheless, $\mathrm{R}^{2}$ values above 0.997 are obtained for the curves relative to the experiments performed at 50 and $60{ }^{\circ} \mathrm{C}$. A better fit for these experiments $\left(R^{2}>0.999\right)$ can be achieved by freeing the value for $n$ in the Gualtieri model, which refines to 1.19 and 1.23 for the experiment at $50{ }^{\circ} \mathrm{C}$ and $60{ }^{\circ} \mathrm{C}$, respectively, with similar values for $a, b$ and $k_{\mathrm{g}}$ to those obtained when $n$ was fixed to 3 (Figure S17). However, these values of $n$ are much less meaningful in physical terms, given that the F4_UiO-66(Ce) phase grows in octahedral shape (Figure 2). Therefore, we preferred to have a slightly worse fit, while preserving a physically sound value of $n$.

Comparing the values of $k_{\mathrm{N}}$ and $k_{\mathrm{g}}$ for F4_MIL-140A(Ce) and F4_UiO-66(Ce) at the same temperatures, we see that F4_UiO-66(Ce) formation is faster both in nucleation and in crystal growth (Table 3, Table S6). Temperature does not seem to have an influence on the relative rates, which stay basically unchanged. Arrhenius analysis for the F4_MIL-140A(Ce) phase shows a similar trend to the one already seen when only $\mathrm{HNO}_{3}$ is present in the reaction environment, with the two fitting lines for nucleation and growth crossing at a $1 / T$ value corresponding to about $54^{\circ} \mathrm{C}$ (Figure 10). The extracted activation energies are $92 \pm 7 \mathrm{~kJ} \mathrm{~mol}^{-1}$ for nucleation and $45 \pm 11 \mathrm{~kJ}$ $\mathrm{mol}^{-1}$ for crystal growth. These values are lower than those found when only $\mathrm{HNO}_{3}$ is present in the reaction environment, but they cannot be straightforwardly compared, due to the presence of a lower amount of $\mathrm{HNO}_{3}$. For the F4_UiO-66(Ce) phase, only the nucleation part could be fitted, finding an activation energy of $94 \pm 15 \mathrm{~kJ}$ $\mathrm{mol}^{-1}$, very similar to that of the F4_MIL-140A(Ce) phase. In the absence of a reliable value of $\mathrm{kg}_{\mathrm{g}}$ for the experiment at $70^{\circ} \mathrm{C}$, no conclusions could be drawn for as concerns crystal growth of the F4_UiO-66(Ce) phase.

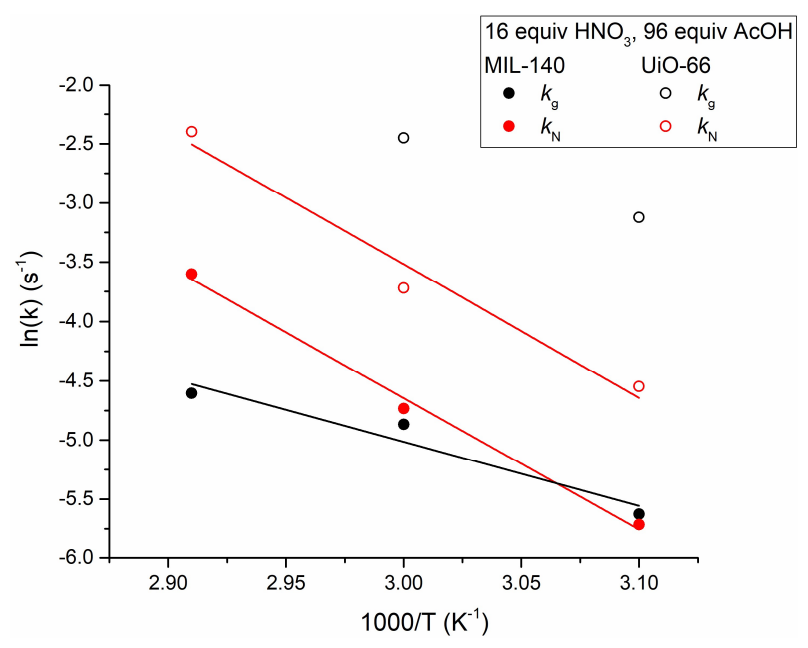

Figure 10. Plot of $\ln \left(k_{N}\right)$ (full red circles) and $\ln \left(k_{\mathrm{g}}\right)$ (full black circles) for F4_MIL-140A(Ce) and $\ln \left(k_{\mathrm{N}}\right)$ (open red circles) and $\ln \left(k_{\mathrm{g}}\right)$ (open black circles) for F4_UiO-66(Ce) versus $1 / \mathrm{T}$ for experiments performed at $50{ }^{\circ} \mathrm{C}, 60^{\circ} \mathrm{C}$ and $70{ }^{\circ} \mathrm{C}$ in the presence of 16 equivalents of $\mathrm{HNO}_{3}$ and 96 equivalents of $\mathrm{AcOH}$. The red line and the black line are the linear Arrhenius fittings for nucleation and crystal growth, respectively. The value of $\ln \left(k_{\mathrm{g}}\right)$ for F4_UiO-66(Ce) at $70{ }^{\circ} \mathrm{C}$ is not displayed because of the non-reliable Gualtieri fitting for this experiment.

\section{Effect of the amount of $\mathrm{HNO}_{3}$}

The second parameter we investigated, as with the first system, was the equivalents of $\mathrm{HNO}_{3}$ used in the reaction. In this case, the amount of $\mathrm{HNO}_{3}$ was varied between 8,12 , and 16 equivalents, whereas temperature was kept constant at $60{ }^{\circ} \mathrm{C}$ and the amount of $\mathrm{AcOH}$ was kept constant at 96 equivalents. In drawing a comparison between the two phases (Figure S18), one can see that the F4_UiO-66(Ce) phase is again kinetically favoured in each experiment. $P_{\mathrm{N}}$ for F4_MIL-140A(Ce) always peaks when crystallisation of F4_UiO-66(Ce) is almost complete, resulting in the two phases not competing for reagents. Again, we can also see some drop in the intensity for the F4_UiO-66(Ce) phase, which suggests that some degradation of this phase takes place over time. Looking at the intensity ratio between the 110 reflection of the F4_MIL-140A(Ce) phase and the 111 
reflection of the F4_UiO-66(Ce) phase at the end of crystallisation, we can see that this increases from 0.6 to 0.8 to 1.3 when $\mathrm{HNO}_{3}$ is increased from 8 to 12 to 16 equivalents, respectively, indicating that a larger amount of $\mathrm{HNO}_{3}$ favours the formation of more $\mathrm{F} 4$ _MIL-140A(Ce). It is to be noted that the largest change in intensity ratio occurs between 12 and 16 equivalents of $\mathrm{HNO}_{3}$, where an almost negligible change in rate constants is observed (vide infra), suggesting that variation of the $\mathrm{HNO}_{3}$ amount within said range mainly has an effect on the equilibrium composition. This could be due to the role of $\mathrm{HNO}_{3}$ in inhibiting deprotonation of $\mathrm{AcOH}$ and its coordination to $\mathrm{Ce}(\mathrm{IV})$ to form the hexanuclear $\left[\mathrm{Ce}_{6} \mathrm{O}_{4}(\mathrm{OH})_{4}(-\mathrm{COO})_{12}\right]$ clusters needed for the F4_UiO-66(Ce) phase. Another possibility could be that lowering the $\mathrm{pH}$ increases the possibility of linker protonation on the formed MOFs themselves, adding additional reversibility to the process and allowing the thermodynamic product F4_MIL-140A(Ce) to be formed, as suggested in a recent paper reporting a systematic investigation of the influence of several parameters on the crystallisation of phase pure MIL-140 type MOFs in DMF solvent. ${ }^{[16]}$ However, the evidence in our hands does not indicate that a more acidic environment promotes such a dissolution-recrystallisation process.

For the F4_MIL-140A(Ce) phase, lowering the equivalents of $\mathrm{HNO}_{3}$ used in the reaction increases the rate of nucleation, with the ratio between 16 and 8 equivalents of 1:15.5 (Figure 11a, Figure S19, Table 4, Table S7). Crystal growth is affected to a similar degree, with a $\mathrm{kg}_{\mathrm{g}}$ ratio of 1:15.2 in the same equivalents range. Growth is rate determining at each amount of $\mathrm{HNO}_{3}$ added, as already observed in the absence of $\mathrm{AcOH}$. The $\mathrm{k}_{\mathrm{N}} / \mathrm{kg}_{\mathrm{g}}$ ratio changes from 1.16 (8 equivalents) to 2.06 (12 equivalents) to 1.14 (16 equivalents), suggesting that the amount of $\mathrm{HNO}_{3}$ does not have a significant effect on the relative rates.
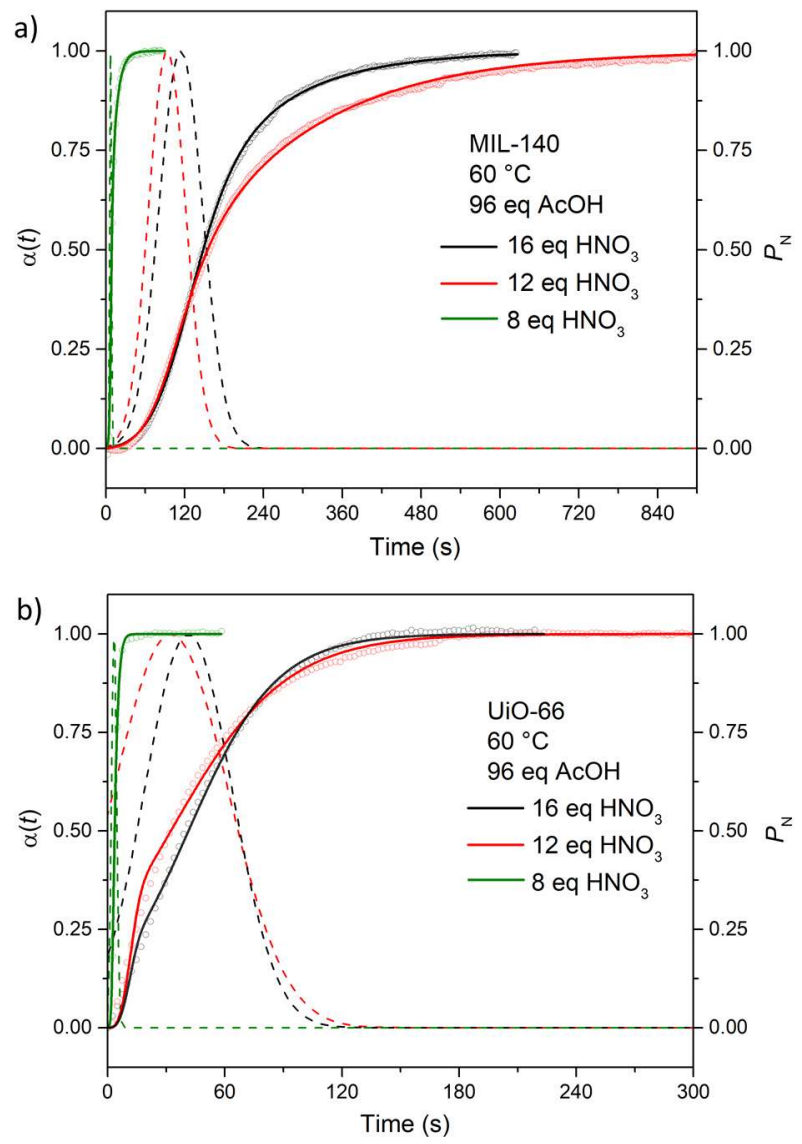

Figure 11. Plot of extent of crystallization ( $\alpha$, empty circles), Gualtieri fitting (solid lines) and probability of nucleation $\left(P_{\mathrm{N}}\right.$, dashed lines) as a function of time for experiments performed at 8 (green), 12 (red) and 16 (black) equivalents of $\mathrm{HNO}_{3}$ for the F4_MIL-140A(Ce) phase (a) and the F4_UiO-66(Ce) phase (b).

Table 4. Rate constants obtained for F4_MIL-140A(Ce) and F4_UiO-66(Ce) in the presence of 8 equivalents $(0.1 \mathrm{~mL}, 0.4 \mathrm{~mol}$ $\left.\mathrm{L}^{-1}\right), 12$ equivalents $\left(0.15 \mathrm{~mL}, 0.6 \mathrm{~mol} \mathrm{~L}^{-1}\right)$, and 16 equivalents $\left(0.2 \mathrm{~mL}, 0.8 \mathrm{~mol} \mathrm{~L}^{-1}\right)$ of $\mathrm{HNO}_{3}$ in the presence of 96 equivalents $\left(1.1 \mathrm{~mL}, 4.8 \mathrm{~mol} \mathrm{~L}^{-1}\right)$ of $\mathrm{AcOH}$ at $60^{\circ} \mathrm{C}$. Full Gualtieri fitting parameters with errors are given in Table S7. 


\begin{tabular}{|c|c|c|c|c|c|c|}
\hline & $\begin{array}{l}\mathrm{HNO}_{3} \\
\text { (eq) }\end{array}$ & $\begin{array}{c}k_{g} \\
\left(s^{-1}\right)\end{array}$ & $\begin{array}{c}\text { Relative } \\
k_{g}\end{array}$ & $\begin{array}{c}k_{N} \\
\left(s^{-1}\right)\end{array}$ & $\begin{array}{c}\text { Relative } \\
k_{N}\end{array}$ & $\begin{array}{l}\text { Ratio } \\
k_{N} / k_{g}\end{array}$ \\
\hline \multirow{3}{*}{ 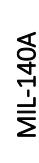 } & 8 & 0.117 & 15.2 & 0.136 & 15.5 & 1.16 \\
\hline & 12 & 0.00524 & 0.7 & 0.01072 & 1.2 & 2.06 \\
\hline & 16 & 0.00769 & 1.0 & 0.00877 & 1.0 & 1.14 \\
\hline \multirow{3}{*}{$\begin{array}{l}\text { ட } \\
\text { 익 }\end{array}$} & 8 & 0.38 & 4.4 & 0.303 & 12.5 & 0.80 \\
\hline & 12 & 0.080 & 0.9 & 0.0309 & 1.3 & 0.39 \\
\hline & 16 & 0.086 & 1.0 & 0.0242 & 1.0 & 0.28 \\
\hline
\end{tabular}

Compared with the F4_MIL-140A(Ce) phase, the effect of lowering the amount of $\mathrm{HNO}_{3}$ in the system is similar on nucleation of the F4_UiO-66(Ce) phase, with a 1:13 $k_{\mathrm{N}}$ ratio between 16 and 8 equivalents (Figure 11b, Figure S20, Table 4, Table S7). The effect on growth is relatively small in comparison, with $k_{\mathrm{g}}$ growing by a factor of 4 in the same $\mathrm{HNO}_{3}$ range. Nucleation is consistently rate determining at each amount of $\mathrm{HNO}_{3}$ added. The $\mathrm{k}_{\mathrm{N}} / \mathrm{kg}_{\mathrm{g}}$ ratio changes from 0.80 (8 equivalents of $\mathrm{HNO}_{3}$ ) to 0.39 (12 equivalents of $\mathrm{HNO}_{3}$ ) to 0.28 (16 equivalents of $\mathrm{HNO}_{3}$ ), suggesting that larger amounts of $\mathrm{HNO}_{3}$ do in fact inhibit nucleation to a larger extent than growth (Table 6 , Table S7). It is to be noted that both $k_{N}$ and $k_{\mathrm{g}}$ stay basically unchanged for both the F4_MIL-140A(Ce) and the F4_UiO-66(Ce) phase when decreasing the amount of $\mathrm{HNO}_{3}$ from 16 to 12 equivalents, suggesting that there is little kinetic effect of $\mathrm{HNO}_{3}$ in this range of parameters, as previously noted. Comparing the values of $k_{\mathrm{N}}$ and $\mathrm{kg}$ for F4_MIL-140A(Ce) and F4_UiO-66(Ce) at the same $\mathrm{HNO}_{3}$ amounts, we see that F4_UiO-66(Ce) formation is consistently faster both in nucleation and in crystal growth (Figure 12, Table S8).

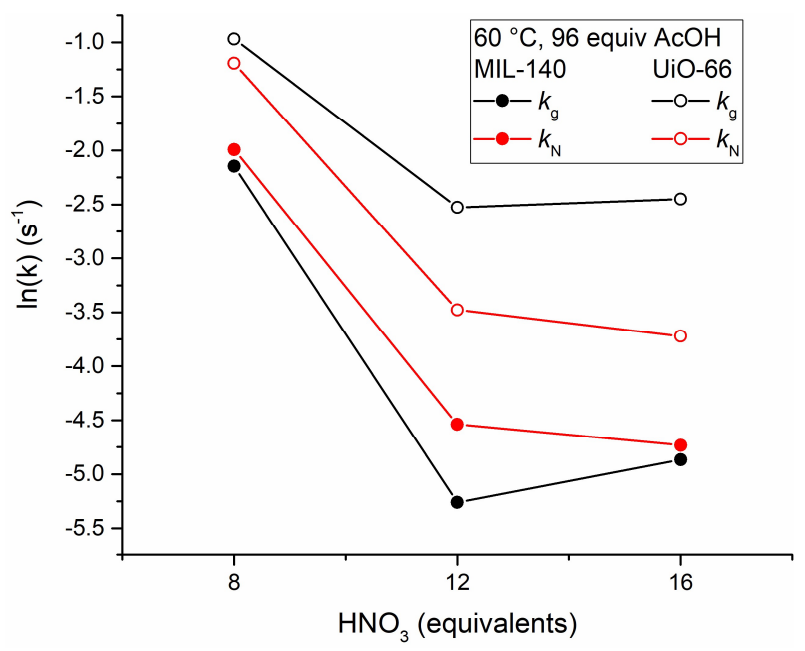

Figure 12. Plot of $\ln \left(k_{\mathrm{N}}\right)$ (full red circles) and $\ln \left(k_{\mathrm{g}}\right)$ (full black circles) for F4_MIL-140A(Ce) and $\ln \left(k_{\mathrm{N}}\right)$ (open red circles) and $\ln \left(k_{\mathrm{g}}\right)$ (open black circles) for $\mathrm{F} 4$ _UiO-66(Ce) versus the amount of $\mathrm{HNO}_{3}$ for experiments performed at $60{ }^{\circ} \mathrm{C}$ and 96 equivalents of $\mathrm{ACOH}$ in the presence of 8,12 and 16 equivalents of $\mathrm{HNO}_{3}$. Lines are a guide for the eye.

\section{Effect of the amount of $\mathrm{AcOH}$}

The third parameter investigated for this system is the amount of $\mathrm{ACOH}$ used, which was varied between 96, 123 and 149 equivalents, while keeping $\mathrm{HNO}_{3}$ fixed at 16 equivalents and temperature fixed at $60{ }^{\circ} \mathrm{C}$. At 149 equivalents of $\mathrm{ACOH}$, the formation F4_MIL-140A(Ce) is very slow and this phase does not reach full crystallisation within the investigated timeframe of $2700 \mathrm{~s}$ (Figure S21). For this reason, we include the result of the experiment performed with 0 equivalents of $\mathrm{AcOH}$ for comparison to identify trends in $k_{\mathrm{N}}$ and $\mathrm{kg}_{\mathrm{g}}$ for the F4_MIL-140A(Ce) phase. Comparing the results of the experiments performed using 96 and 123 equivalents of $\mathrm{AcOH}$, we note that increasing the amount of $\mathrm{AcOH}$ leads to slowing down the crystallisation rate for both phases (Figure S22). Once again, the F4_UiO-66(Ce) phase is clearly kinetically favoured and $P_{\mathrm{N}}$ for F4_MIL-140A(Ce) peaks when crystallisation of F4_UiO-66(Ce) is almost complete, resulting in the two phases not competing for reagents (Figure S22). We can also see slow degradation of the F4_UiO-66(Ce) phase over time. A decrease in 
the ratio between the intensity of the F4_MIL-140A(Ce) 110 reflection and the F4_UiO-66(Ce) 111 reflection from 1.3 to 0.9 is found when $\mathrm{AcOH}$ increases from 96 to 123 equivalents. This suggests that $\mathrm{AcOH}$ shifts the equilibrium in favour of the formation of F4_UiO-66(Ce) over F4_MIL-140A(Ce) and that the coordination modulator favours indeed the kinetic product. This is probably due to the role of $\mathrm{AcOH}$ in promoting the formation of the hexanuclear $\left[\mathrm{Ce}_{6} \mathrm{O}_{4}(\mathrm{OH})_{4}(-\mathrm{COO})_{12}\right]$ clusters needed for the F4_UiO-66(Ce) phase, thus reducing the availability of isolated metal ions that can react with $\mathrm{TFBDC}^{2-}$ in solution to form the extended inorganic building units at the basis of F4_MIL-140A(Ce).
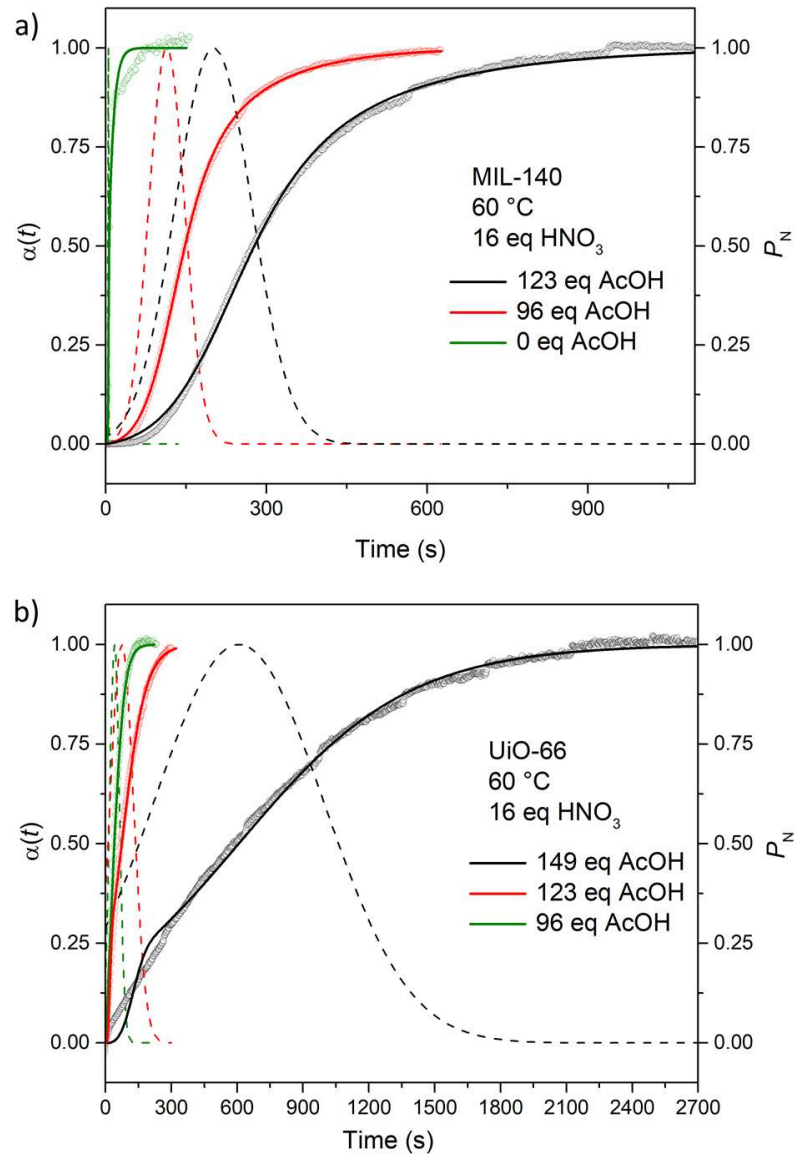

Figure 13. Plot of extent of crystallization ( $\alpha$, empty circles), Gualtieri fitting (solid lines) and probability of nucleation $\left(P_{\mathrm{N}}\right.$, dashed lines) as a function of time for experiments performed with 16 equivalents of $\mathrm{HNO}_{3}$ at $60{ }^{\circ} \mathrm{C}$ and with 0,96 , and 123 equivalents of $\mathrm{ACOH}$ and 16 equivalents of $\mathrm{HNO}_{3}$ at $60{ }^{\circ} \mathrm{C}$ for the $\mathrm{F} 4$ _MIL-140A(Ce) phase (a) and with 96, 123, and 149 equivalents of $\mathrm{ACOH}$ for the F4_UiO-66(Ce) phase (b).

We see here that decreasing the amount of $\mathrm{AcOH}$ present in the system has a relatively similar effect on nucleation and crystal growth of the F4_MIL-140A(Ce) phase, which increase by a factor of 38.7 and 27.8, respectively, when AcOH goes from 123 to 0 equivalents (Figure 13a, Figure S23, Table 5, Table S9). Crystal growth is rate determining in all the range investigated. The $\mathrm{kN} / \mathrm{kg}$ ratio changes from 1.68 ( 0 equivalents) to 1.14 (96 equivalents) to 1.21 (123 equivalents), suggesting that the amount of $\mathrm{AcOH}$ has a similar effect on the nucleation and crystal growth stages. 
Table 5. Rate constants obtained for F4_MIL-140A(Ce) and F4_UiO-66(Ce) in the presence of 0 equivalents, 96 equivalents $\left(1.1 \mathrm{~mL}, 4.8 \mathrm{~mol} \mathrm{~L}^{-1}\right), 123$ equivalents $\left(1.4 \mathrm{~mL}, 6.1 \mathrm{~mol} \mathrm{~L}^{-1}\right)$, and 149 equivalents $(1.7 \mathrm{~mL}, 7.4 \mathrm{~mol} \mathrm{~L}-1)$ of $\mathrm{AcOH}$, and 16 equivalents $\left(0.2 \mathrm{~mL}, 0.8 \mathrm{~mol} \mathrm{~L}^{-1}\right)$ of $\mathrm{HNO}_{3}$ at $60{ }^{\circ} \mathrm{C}$. Full Gualtieri fitting parameters with errors are given in Table $\mathrm{S} 9$.

\begin{tabular}{|c|c|c|c|c|c|c|}
\hline & $\begin{array}{c}\mathrm{AcOH} \\
\text { (eq) }\end{array}$ & $\begin{array}{c}k_{g} \\
\left(\mathbf{s}^{-1}\right)\end{array}$ & $\begin{array}{c}\text { Relative } \\
k_{g}\end{array}$ & $\begin{array}{c}k_{N} \\
\left(s^{-1}\right)\end{array}$ & $\begin{array}{c}\text { Relative } \\
k_{N}\end{array}$ & $\begin{array}{l}\text { Ratio } \\
k_{N} / k_{g}\end{array}$ \\
\hline \multirow{3}{*}{ 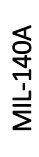 } & 0 & 0.114 & 27.8 & 0.19 & 38.7 & 1.7 \\
\hline & 96 & 0.00769 & 1.9 & 0.00877 & 1.8 & 1.1 \\
\hline & 123 & 0.00401 & 1.0 & 0.00498 & 1.0 & 1.2 \\
\hline \multirow{3}{*}{ 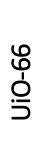 } & 96 & 0.086 & 11.5 & 0.0242 & 14.7 & 0.28 \\
\hline & 123 & 0.047 & 6.3 & 0.0135 & 8.2 & 0.29 \\
\hline & 149 & 0.0075 & 1.0 & 0.001647 & 1.0 & 0.22 \\
\hline
\end{tabular}

Regarding the effect of decreasing $\mathrm{AcOH}$ on the crystallisation of F4_UiO-66(Ce), there is a similar increase in the rates of nucleation and crystal growth, which grow by a factor of 14.7 and 11.5, respectively, between 149 and 96 equivalents of $\mathrm{AcOH}$ (Figure 13b, Figure S24, Table 5, Table S9). Nucleation is consistently rate determining at each amount of $\mathrm{AcOH}$ added. The $\mathrm{kN} / \mathrm{kg}$ ratio changes from 0.28 (96 equivalents of $\mathrm{AcOH}$ ) to 0.29 (123 equivalents of $\mathrm{AcOH}$ ) to 0.22 (149 equivalents of $\mathrm{AcOH}$ ), suggesting that $\mathrm{AcOH}$ affects nucleation and growth to the same extent. Comparing the values of $k_{\mathrm{N}}$ and $k_{\mathrm{g}}$ for F4_MIL-140A(Ce) and F4_UiO-66(Ce) at the same AcOH amounts, we see that F4_UiO-66(Ce) formation is faster both in nucleation and in growth (Figure 14, Table S10).

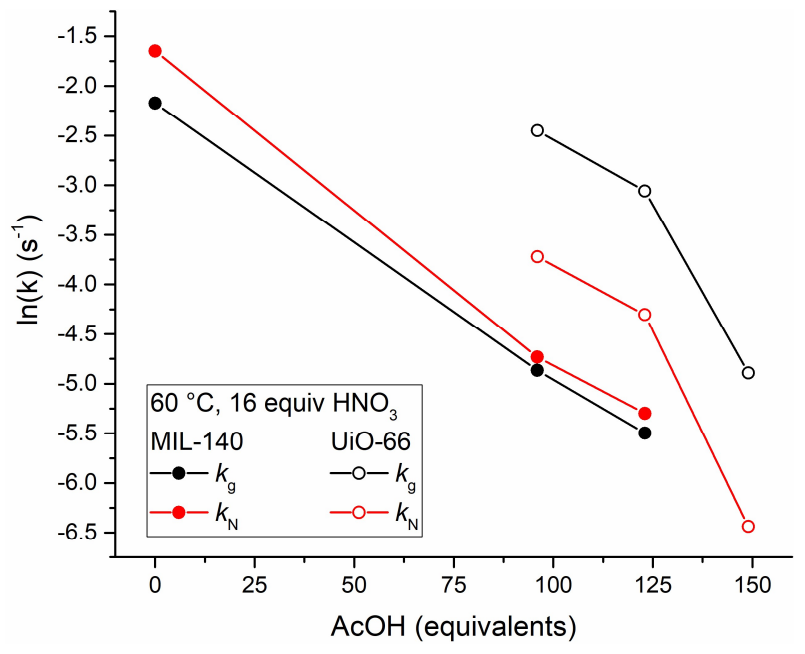

Figure 14. Plot of $\ln \left(k_{N}\right)$ (full red circles) and $\ln \left(k_{\mathrm{g}}\right)$ (full black circles) for F4_MIL-140A(Ce) and $\ln \left(k_{N}\right)$ (open red circles) and $\ln \left(k_{\mathrm{g}}\right)$ (open black circles) for F4_UiO-66(Ce) versus the amount of AcOH for experiments performed at $60{ }^{\circ} \mathrm{C}$ and 16 equivalents of $\mathrm{HNO}_{3}$ in the presence of $0,96,123$ and 149 equivalents of $\mathrm{AcOH}$.

\section{System III: phase pure F4_UiO-66(Ce)}

In order to achieve the pure F4_UiO-66(Ce) phase, the conditions require the largest amount of $\mathrm{AcOH}$ investigated so far (149 equivalents), with some $\mathrm{HNO}_{3}$ (8 equivalents) present in order to slow down the kinetics, which allows for crystallisation to be followed in situ. We kept the concentration of CAN and $\mathrm{H}_{2}$ TFBDC fixed at $0.05 \mathrm{~mol} \mathrm{~L}^{-1}$ and investigated the effect of temperature on this pure F4_UiO-66(Ce) phase, running the reactions at 60,70 , and $80^{\circ} \mathrm{C}$. Full crystallisation is achieved in less than five minutes in all experiments, with the rate increasing when moving from 60 to 70 to $80^{\circ} \mathrm{C}$ (Figure 15). It should be noted that, in this case, the $n$ value was not specifically determined but left free to refine due to the lack of convergence of the fit when $n$ was constrained to 3 (Figure S25). However, all the fittings end up with a similar value for $n(\sim 0.5)$, despite the lack of constraint, which could still allow us to make meaningful comparisons in this dataset and in turn draw conclusions on the kinetics of the experiment, even if the physical meaning of the parameter is lost. Contrary to 
what has been seen in previous experiments for the F4_UiO-66(Ce) phase, crystal growth seems to be rate determining in this case (Table 6, Table S11). Nucleation is practically instantaneous and $P_{\mathrm{N}}$ peaks within $5 \mathrm{~s}$ in each experiment (Figure 16). Quite surprisingly, $k_{\mathrm{N}}$ decreases when the temperature is increased above $60^{\circ} \mathrm{C}$. However, given the extremely fast nucleation process, we have a small number of observables in the timeframe where nucleation occurs, introducing significant errors in the fitting (see Table S11). Furthermore, we suspect that the temperature drop following addition of the CAN solution (held at RT) might affect the experiments performed at higher temperature to a larger extent, which could also explain the counterintuitive results obtained for $k_{\mathrm{N}}$. Arrhenius analysis of the crystal growth stage gives an activation energy of $67 \pm 4 \mathrm{~kJ} \mathrm{~mol}^{-1}$ (Figure 16), a value similar to the one extracted for the F4_MIL-140A(Ce) phase in a similar temperature range and in the presence of 32 equivalents of $\mathrm{HNO}_{3}$ (Figure 5).

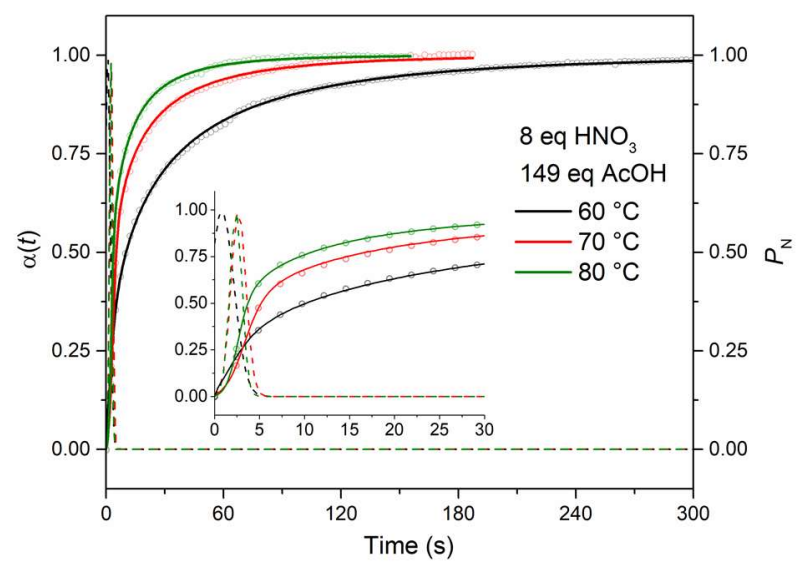

Figure 15. Plot of extent of crystallization ( $\alpha$, empty circles), Gualtieri fitting (solid lines) and probability of nucleation $\left(P_{\mathrm{N}}\right.$, dashed lines) as a function of time for experiments performed at $60^{\circ} \mathrm{C}$ (black), $70^{\circ} \mathrm{C}$ (red) and $80^{\circ} \mathrm{C}$ (green) in the presence of 8 equivalents of $\mathrm{HNO}_{3}$ and 149 equivalents of $\mathrm{AcOH}$ for the $\mathrm{F} 4$ _UiO-66(Ce) phase.

Table 6. Rate constants obtained for F4_UiO-66(Ce) in the presence of 8 equivalents $\left(0.1 \mathrm{~mL}, 0.4 \mathrm{~mol} \mathrm{~L}^{-1}\right)$ of $\mathrm{HNO}_{3}$, and 149 equivalents $\left(1.7 \mathrm{~mL}, 7.4 \mathrm{~mol} \mathrm{~L}^{-1}\right)$ of $\mathrm{AcOH}$ at 50,60 , and $70^{\circ} \mathrm{C}$. Full Gualtieri fitting parameters with errors are given in Table S11.

\begin{tabular}{cccccc}
\hline $\begin{array}{c}\text { Temp } \\
\left({ }^{\circ} \mathbf{C}\right)\end{array}$ & $\begin{array}{c}\boldsymbol{k}_{g} \\
\left(\mathbf{s}^{-1}\right)\end{array}$ & $\begin{array}{c}\text { Relative } \\
\boldsymbol{k}_{\boldsymbol{g}}\end{array}$ & $\begin{array}{c}\boldsymbol{k}_{N} \\
\left(\mathbf{s}^{-1}\right)\end{array}$ & $\begin{array}{c}\text { Relative } \\
\boldsymbol{k}_{N}\end{array}$ & $\begin{array}{c}\text { Ratio } \\
\boldsymbol{k}_{N} / \boldsymbol{k}_{\boldsymbol{g}}\end{array}$ \\
\hline $\mathbf{8 0}$ & 0.194 & 3.9 & 0.422 & 0.4 & 2.17 \\
$\mathbf{7 0}$ & 0.112 & 2.2 & 0.37 & 0.3 & 3.36 \\
$\mathbf{6 0}$ & 0.0498 & 1.0 & 1.3 & 1.0 & 24.10 \\
\hline
\end{tabular}




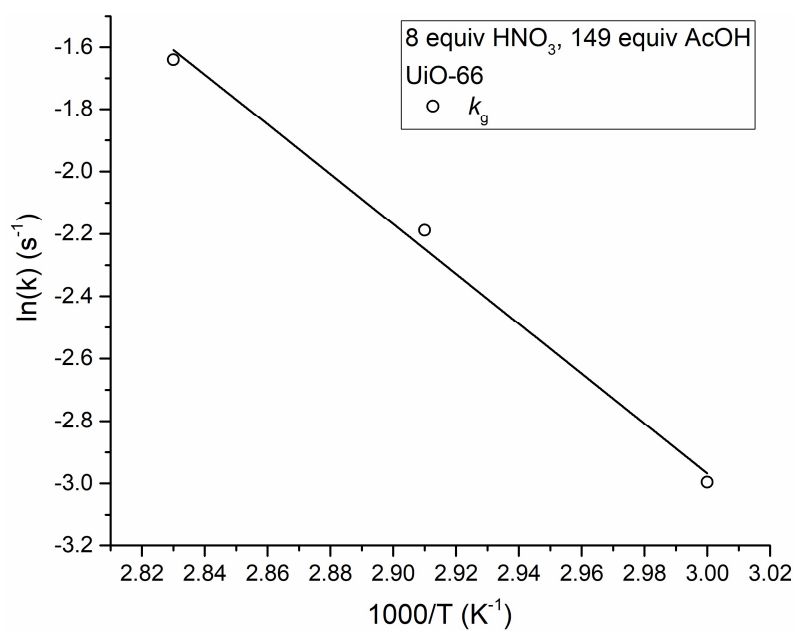

Figure 16. Plot of $\ln \left(k_{\mathrm{g}}\right)$ (open black circles) for F4_UiO-66(Ce) versus $1 / \mathrm{T}$ for experiments performed at $60{ }^{\circ} \mathrm{C}, 70{ }^{\circ} \mathrm{C}$ and 80 ${ }^{\circ} \mathrm{C}$ in the presence of 8 equivalents of $\mathrm{HNO}_{3}$ and 149 equivalents of $\mathrm{AcOH}$. The black line is the linear Arrhenius fitting.

\section{Ex situ optimisation of F4_MIL-140A(Ce) synthesis}

Having gathered precious information about the kinetics of crystallisation and how these are affected by various reaction parameters during the in situ study, we carried out further synthetic work to investigate the possibility to expand the range of different $\mathrm{Ce}(\mathrm{IV})$ precursors as well as alternative inorganic acid modulators, with the aim of identifying different optimised protocols for the prospective production of F4_MIL-140A(Ce) on a larger scale. We first performed a series of experiments to look at the effect of temperature on the yield and product quality when $\mathrm{HNO}_{3}$ was kept at 32 equivalents. We deemed the synthesis performed at $60{ }^{\circ} \mathrm{C}$ an optimal compromise between temperature, reaction time, quality of the product and yield. We successively looked at using a different protonation modulator, employing $\mathrm{H}_{2} \mathrm{SO}_{4}$ in place of $\mathrm{HNO}_{3}$ in otherwise identical conditions. We found that a much lower amount of $\mathrm{H}_{2} \mathrm{SO}_{4}$ is needed to effectively modulate crystallisation kinetics, affording a lower quality product than $\mathrm{HNO}_{3}$. However, $\mathrm{H}_{2} \mathrm{SO}_{4}$ significantly reduces the impact of the parallel redox process. Finally, we evaluated the effect of using $\mathrm{Ce}\left(\mathrm{SO}_{4}\right)_{2}$ as a metal precursor in the presence of either $\mathrm{HNO}_{3} \mathrm{Or} \mathrm{H}_{2} \mathrm{SO}_{4}$ as protonation modulator, finding that this affords lower quality product than CAN. Further details on the optimisation work are provided in the SI (Figures S26-29).

These experiments demonstrate that the combination of CAN as the $\mathrm{Ce}$ (IV) precursor and $\mathrm{HNO}_{3}$ as the protonation modulator provides the best compromise in terms of reaction time, yield and quality of the F4_MIL$140 \mathrm{~A}(\mathrm{Ce})$ product. This material displays the characteristic phase-change behaviour expected for F4_MIL$140 \mathrm{~A}(\mathrm{Ce})$ and even higher $\mathrm{CO}_{2}$ uptake at saturation (about $2.6 \mathrm{mmol} \mathrm{g}{ }^{-1}$, Figures $\mathrm{S} 30-31$ ) than that previously observed for the MOF obtained with the original synthesis conditions (about $2.0 \mathrm{mmol} \mathrm{g}$ ). ${ }^{31}$ Therefore, future efforts will be aimed at upscaling the synthetic protocol using $\mathrm{CAN}$ and $\mathrm{HNO}_{3}$. The fact that the reaction temperature $\left(60^{\circ} \mathrm{C}\right)$ is well below the normal boiling point of water is a great advantage for upscaling, because there is no need for sophisticated, pressure resistant vessels.

\section{Conclusions}

In this work, we have performed a systematic in-situ PXRD investigation of the influence of various reaction parameters on the thermodynamics and kinetics of crystallisation of the recently discovered perfluorinated $\mathrm{Ce}$ (IV)-based MOFs F4_MIL-140A(Ce) and F4_UiO-66(Ce) in aqueous medium. We introduced the use of $\mathrm{HNO}_{3}$ as a protonation modulator, which allowed us to finely control the kinetics of crystallisation. The main outcomes of this investigation are visually summarised in Figure $\mathrm{S} 32$. In the presence of just $\mathrm{HNO}_{3}$, phase pure F4_MIL$140 \mathrm{~A}(\mathrm{Ce})$ was observed (System I in Figure S32). The effect of temperature and amount of $\mathrm{HNO}_{3}$ was assessed for this system, finding that heating mainly accelerates nucleation, whereas the modulator influences nucleation and crystal growth to a similar extent. At temperatures above $57{ }^{\circ} \mathrm{C}$, crystal growth becomes the rate determining process. Upon addition of $\mathrm{AcOH}$ to the system, alongside $\mathrm{HNO}_{3}$, mixed-phased products, consisting of F4_MIL-140A(Ce) and F4_UiO-66(Ce), were obtained (System II in Figure S32). This makes it clear that AcOH is essential for the formation of the F4_UiO-66(Ce) phase. In all experiments with AcOH, the F4_UiO-66(Ce) 
phase formed earlier and faster than the F4_MIL-140A(Ce) phase, but we did not observe any significant degree of interconversion between the two phases in the investigated reaction conditions, suggesting that the two phases form independently and compete for the reagents in solution. Different from F4_MIL-140A(Ce), crystal growth is the rate determining step for the F4_UiO-66(Ce) phase. Comparing the two modulators in the mixedphase system, we see that a decrease in the amount of $\mathrm{HNO}_{3}$ present results in the preferential acceleration of nucleation for the F4_UiO-66(Ce) phase, whereas the F4_MIL-140A(Ce) phase experiences similar effect on nucleation and crystal growth. For $\mathrm{AcOH}$, a decrease in the amount used leads to the acceleration of both nucleation and growth to a similar extent for both phases. Looking at the intensity ratios of the fully crystallised products alone, it is clear that $\mathrm{HNO}_{3}$ favours the formation of the F4_MIL-140A(Ce) phase, while AcOH favours the formation of the F4_UiO-66(Ce) phase, which can be obtained in pure form in the presence of a large amount of $\mathrm{AcOH}$ (149 equivalents) and a little $\mathrm{HNO}_{3}$ (8 equivalents). Thus, the coordination modulator appears to favour the kinetic product, probably by promoting the formation of the inorganic clusters necessary to construct the framework of F4_UiO-66(Ce), which grows upon exchange of acetate by TFBDC ${ }^{2-}$. This process reduces the concentration of isolated $\mathrm{Ce}(\mathrm{IV})$ ions from the solution, preventing them from reacting with $\mathrm{TFBDC}^{2-}$ and form F4_MIL-140A(Ce). Finally, we screened different combinations of $\mathrm{Ce}(\mathrm{IV})$ source and protonation modulator for the synthesis of F4_MIL-140A(Ce) ex situ, finding that the best results are obtained when CAN is the metal source and $\mathrm{HNO}_{3}$ is the modulator. Based on these results, an optimised route to achieving F4_MIL-140A(Ce) in mild conditions $\left(60^{\circ} \mathrm{C}, 1 \mathrm{~h}\right)$ at the small scale was identified. The fundamental understanding of the crystallisation process of perfluorinated $\mathrm{Ce}(\mathrm{IV})$-based MOFs gained during this work will be crucial to develop protocols for the scale up of their synthesis, in particular for F4_MIL-140A(Ce), given its interesting $\mathrm{CO}_{2}$ capture properties.

\section{Acknowledgments}

Part of this research was carried out at P23/PETRAIII at DESY, a member of the Helmholtz Association (HGF) (Proposal I-20190218 EC). The research leading to this result has been supported by the project CALIPSOplus under the Grant Agreement 730872 from the EU Framework Programme for Research and Innovation HORIZON2020. The authors thank Timo Rabe (CAU Kiel) for support during installation of the SynRAC unit, Dr Valentina Crocellà (University of Torino) for collecting the $\mathrm{N}_{2}$ adsorption isotherm and Carla Nicola for handdrawing the graphical abstract. The authors acknowledge the European Union's Horizon 2020 research and innovation programme under the Marie Skłodowska-Curie grant agreement no 663830 (M. T.). Financial support was also provided by the FLEXIS and Reducing Industrial Carbon Emissions (RICE) research operations part funded by the EU's European Regional Development Fund through the Welsh Government. The authors acknowledge the assistance provided by the Swansea University AIM Facility, which was funded in part by the EPSRC EP/M028267/1, the ERDF through the Welsh Government grant 80708, and the Sêr Solar project via the Welsh Government.

\section{References}

[1] a) M. J. Van Vleet, T. Weng, X. Li, J. R. Schmidt, Chem. Rev. 2018, 118, 3681-3721; b) R. I. Walton, F. Millange, in The Chemistry of Metal-Organic Frameworks: Synthesis, Characterization, and Applications (Ed.: S. Kaskel), Wiley-VCH, 2016, pp. 729-764; c) N. Pienack, W. Bensch, Angew. Chem. Int. Ed. 2011, 50, 2014-2034.

[2] a) H. H. Yeung, A. F. Sapnik, F. Massingberd-Mundy, M. W. Gaultois, Y. Wu, D. A. X. Fraser, S. Henke, R. Pallach, N. Heidenreich, O. V. Magdysyuk, N. T. Vo, A. L. Goodwin, Angew. Chem. Int. Ed. 2019, 58, 566-571; b) Y. Wu, M. I. Breeze, G. J. Clarkson, F. Millange, D. O'Hare, R. I. Walton, Angew. Chem. Int. Ed. 2016, 55, 4992-4996; c) G. Zahn, P. Zerner, J. Lippke, F. L. Kempf, S. Lilienthal, C. A. Schröder, A. M. Schneider, P. Behrens, CrystEngComm 2014, 16, 9198-9207; d) J. Cravillon, C. A. Schröder, H. Bux, A. Rothkirch, J. Caro, M. Wiebcke, CrystEngComm 2012, 14, 492-498; e) F. Millange, R. El Osta, M. E. Medina, R. I. Walton, CrystEngComm 2011, 13, 103-108.

[3] a) X. Sang, J. Zhang, J. Xiang, J. Cui, L. Zheng, J. Zhang, Z. Wu, Z. Li, G. Mo, Y. Xu, J. Song, C. Liu, X. Tan, T. Luo, B. Zhang, B. Han, Nat. Commun. 2017, 8, 175; b) M. G. Goesten, E. Stavitski, J. Juan-Alcañiz, A. Martiñez-Joaristi, A. V. Petukhov, F. Kapteijn, J. Gascon, Catal. Today 2013, 205, 120-127; c) E. Stavitski, M. Goesten, J. Juan-Alcaniz, A. Martinez-Joaristi, P. Serra-Crespo, A. V. Petukhov, J. Gascon, F. Kapteijn, Angew. Chem. Int. Ed. 2011, 50, 9624-9628.

[4] a) S. Van Cleuvenbergen, Z. J. Smith, O. Deschaume, C. Bartic, S. Wachsmann-Hogiu, T. Verbiest, M. A. van der Veen, Nat. Commun. 2018, 9, 3418; b) J. Cravillon, R. Nayuk, S. Springer, A. Feldhoff, K. Huber, M. Wiebcke, Chem. Mater. 2011, 23, 21302141

[5] C. L. Jones, C. E. Hughes, H. H. M. Yeung, A. Paul, K. D. M. Harris, T. L. Easun, Chemical Science 2021.

[6] a) S. L. Griffin, M. L. Briuglia, J. H. Ter Horst, R. S. Forgan, Chem. Eur. J. 2020, 26, 6910-6918; b) F. Massingberd-Mundy, S. Poulston, S. Bennett, H. H. Yeung, T. Johnson, Sci. Rep. 2020, 10, 17355

[7] a) B. Bueken, H. Reinsch, N. Heidenreich, A. Vandekerkhove, F. Vermoortele, C. E. A. Kirschhock, N. Stock, D. De Vos, R. Ameloot, CrystEngComm 2017, 19, 4152-4156; b) H. H. Yeung, Y. Wu, S. Henke, A. K. Cheetham, D. O'Hare, R. I. Walton, Angew. Chem. Int. 
Ed. 2016, 55, 2012-2016; c) Y. Wu, S. Henke, G. Kieslich, I. Schwedler, M. Yang, D. A. X. Fraser, D. O'Hare, Angew. Chem. 2016, 128, 14287-14290; d) M. Feyand, A. Hubner, A. Rothkirch, D. S. Wragg, N. Stock, Inorg. Chem. 2012, 51, 12540-12547.

[8] a) Y. F. Zhang, Q. Wang, D. X. Xue, J. Bai, Inorg. Chem. 2020, 59, 11233-11237; b) M. Lammert, C. Glißmann, H. Reinsch, N. Stock, Cryst. Growth Des. 2017, 17, 1125-1131; c) M. Lammert, H. Reinsch, C. A. Murray, M. T. Wharmby, H. Terraschke, N. Stock, Dalton Trans. 2016, 45, 18822-18826; d) M. Lammert, M. T. Wharmby, S. Smolders, B. Bueken, A. Lieb, K. A. Lomachenko, D. D. Vos, N. Stock, Chem. Commun. 2015, 51, 12578-12581.

[9] a) S. Smolders, K. A. Lomachenko, B. Bueken, A. Struyf, A. L. Bugaev, C. Atzori, N. Stock, C. Lamberti, M. B. J. Roeffaers, D. E. De Vos, ChemPhysChem 2018, 19, 373-378; b) T. Islamoglu, A. Atilgan, S.-Y. Moon, G. W. Peterson, J. B. DeCoste, M. Hall, J. T. Hupp, O. K. Farha, Chem. Mater. 2017, 29, 2672-2675.

[10] a) Y. Song, Y. Pi, X. Feng, K. Ni, Z. Xu, J. S. Chen, Z. Li, W. Lin, J. Am. Chem. Soc. 2020, 142, 6866-6871; b) M. Campanelli, T. Del Giacco, F. De Angelis, E. Mosconi, M. Taddei, F. Marmottini, R. D'Amato, F. Costantino, ACS Appl. Mater. Interfaces. 2019, 11, 45031-45037; c) X. Qiu, Y. Zhu, X. Zhang, Y. Zhang, L. T. Menisa, C. Xia, S. Liu, Z. Tang, Sol. RRL 2019, 4; d) X. P. Wu, L. Gagliardi, D. G. Truhlar, J. Am. Chem. Soc. 2018, 140, 7904-7912.

[11] J. Jacobsen, A. lenco, R. D'Amato, F. Costantino, N. Stock, Dalton Trans. 2020.

[12] N. Heidenreich, S. Waitschat, H. Reinsch, Z. Anorg. Allg. Chem. 2018, 644, 1826-1831.

[13] R. D'Amato, A. Donnadio, M. Carta, C. Sangregorio, D. Tiana, R. Vivani, M. Taddei, F. Costantino, ACS Sustainable Chem. Eng. $2018,7,394-402$.

[14] V. Guillerm, F. Ragon, M. Dan-Hardi, T. Devic, M. Vishnuvarthan, B. Campo, A. Vimont, G. Clet, Q. Yang, G. Maurin, G. Ferey, A. Vittadini, S. Gross, C. Serre, Angew. Chem. Int. Ed. 2012, 51, 9267-9271.

[15] a) D. Bara, C. Wilson, M. Mortel, M. M. Khusniyarov, S. Ling, B. Slater, S. Sproules, R. S. Forgan, J Am Chem Soc 2019, 141, 83468357; b) L. Yang, T. Zhao, I. Boldog, C. Janiak, X. Y. Yang, Q. Li, Y. J. Zhou, Y. Xia, D. W. Lai, Y. J. Liu, Dalton Trans 2019, 48, 989-996.

[16] M. Schulz, N. Marquardt, M. Schafer, D. P. Warwas, S. Zailskas, A. Schaate, Chemistry 2019, 25, 13598-13608.

[17] N. Heidenreich, U. Rutt, M. Koppen, A. K. Inge, S. Beier, A. C. Dippel, R. Suren, N. Stock, Rev. Sci. Instrum. 2017, 88, 104102.

[18] a) J. Filik, A. W. Ashton, P. C. Y. Chang, P. A. Chater, S. J. Day, M. Drakopoulos, M. W. Gerring, M. L. Hart, O. V. Magdysyuk, S. Michalik, A. Smith, C. C. Tang, N. J. Terrill, M. T. Wharmby, H. Wilhelm, J. Appl. Crystallogr. 2017, 50, 959-966; b) M. Basham, J. Filik, M. T. Wharmby, P. C. Chang, B. El Kassaby, M. Gerring, J. Aishima, K. Levik, B. C. Pulford, I. Sikharulidze, D. Sneddon, M. Webber, S. S. Dhesi, F. Maccherozzi, O. Svensson, S. Brockhauser, G. Naray, A. W. Ashton, J. Synchrotron Radiat. 2015, 22, 853858.

[19] A. F. Gualtieri, Phys. Chem. Miner. 2001, 28, 719-728

[20] S. J. Gurgul, G. Seng, G. R. Williams, J. Synchrotron Radiat. 2019, 26, 774-784.

[21] H. Reinsch, N. Stock, CrystEngComm 2013, 15, 544-550.

[22] F. Ragon, P. Horcajada, H. Chevreau, Y. K. Hwang, U. H. Lee, S. R. Miller, T. Devic, J. S. Chang, C. Serre, Inorg. Chem. 2014, 53, 2491-2500. 
TABLE OF CONTENTS

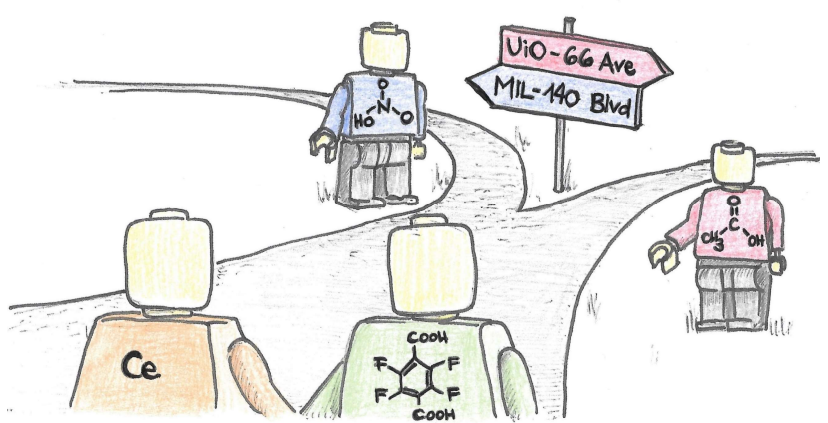

In situ synchrotron powder $\mathrm{X}$-ray diffraction is employed to investigate the crystallisation of perfluorinated $\mathrm{Ce}$ (IV)-based metal-organic frameworks with UiO-66 and MIL-140 topology in aqueous environment. The effects of reaction temperature, $\mathrm{HNO}_{3}$ as protonation modulator and $\mathrm{CH}_{3} \mathrm{COOH}$ as coordination modulator on the kinetics and thermodynamics of the process are evaluated. 